\title{
Variação da Pressão Sistólica como Indicadora Precoce de Hipovolemia e Guia de Reposição Volêmica com Solução Hiperosmótica e Hiperoncótica no Cão *
}

\section{Systolic Pressure Variation as an Earlier Hypovolemia Indicator and a Guide for Volume Replacement with Hypertonic and Hyperoncotic Solution in Dogs}

\author{
Odilar de Paiva Filho ${ }^{1}$, José Reinaldo Cerqueira Braz, TSA ${ }^{2}$, Fredson de Paula e Silva ${ }^{3}$,
} Tiago Otávio Pedro ${ }^{3}$, Paulo do Nascimento Júnior, TSA ${ }^{4}$

\begin{abstract}
RESUMO
Paiva Filho O, Braz JRC, Silva FP, Pedro TO, Nascimento Jr P - Variação da Pressão Sistólica como Indicadora Precoce de Hipovolemia e Guia de Reposição Volêmica com Solução Hiperosmótica e Hiperoncótica no Cão

JUSTIFICATIVA E OBJETIVOS: Estudos introduziram novo método para avaliação da pré-carga, baseado na análise da variação da pressão sistólica (VPS) durante ventilação artificial. O objetivo desta pesquisa é avaliar se a VPS e sua derivada delta down (ddown) são indicadoras precoces de hipovolemia e guias de reposição volêmica com solução hiperosmótica e hiperoncótica. MÉTODO: Doze cães foram submetidos a sangramentos parciais de $5 \%$ da volemia até se atingir $20 \%$ da volemia $\left(14 \mathrm{ml} . \mathrm{kg}^{-1}\right)$. Antes (controle) e após cada sangramento foram realizadas análises hemodinâmicas, respiratórias e sangüíneas. Após, os cães foram submetidos à reposição com solução de $\mathrm{NaCl}$ a $7,5 \%$ em dextran 70 a $3,75 \%$ (SHD) (4 ml. $\left.\mathrm{kg}^{-1}\right)$ e novas análises dos atributos estudados foram realizadas aos 5 e 30 minutos após a reposição.

RESULTADOS: A pressão arterial média diminuiu durante o sangramento e aumentou após a reposição, sem retornar aos valores do controle. As pressões da artéria pulmonar e do átrio direito $(P A D)$ diminuíram antes e aumentaram após a reposição para valores semelhantes aos do controle. A pressão da artéria pulmonar ocluída (PAPO) diminuiu após o primeiro sangramento e manteve-se em valores abaixo aos do controle, mesmo após a reposição. O índice cardíaco não se alterou, mas aumentou após a reposição, para valores superiores aos do controle. O índice sistólico (IS) diminuiu antes e aumentou após a reposição, em níveis superiores aos do controle. Os índices de resistência vascular sistêmica (IRVS) e pulmonar (IRVP) não se alteraram antes, mas diminuíram após a reposição, com o IRVS em níveis inferiores aos do controle, e o IRVP em níveis semelhantes aos do controle. Os índices de trabalho sistólico dos ventrículos direito (ITSVD) e esquerdo (ITSVE) diminuíram durante o sangramento, mas aumentaram após a reposição, com o ITSVD em níveis superiores aos do controle e o ITSVE em níveis semelhantes aos do controle. A VPS e ddown aumentaram progressivamente durante $O$
\end{abstract}

\footnotetext{
* Recebido do (Received from) Laboratório Experimental do CET/SBA do Departamento de Anestesiologia da Faculdade de Medicina de Botucatu (FMB-UNESP)

1. Pós-Graduando (Mestrado) do Programa de Pós-Graduação em Anestesiologia da FMB-UNESP. Bolsista CAPES

2. Professor Titular do CET/SBA do Depto de Anestesiologia

3. Graduando da FMB-UNESP. Bolsista do Programa PIBIC/CNPq

4. Professor Assistente Doutor do CET/SBA do Depto de Anestesiologia

Endereço para correspondência (Correspondence to)

Dr. José Reinaldo Cerqueira Braz

Dept $^{\circ}$ de Anestesiologia da FMB-UNESP

18618-970 Botucatu, SP
}

Apresentado (Submitted) em 18 de setembro de 2002

Aceito (Accepted) para publicação em 17 de outubro de 2002

(C) Sociedade Brasileira de Anestesiologia, 2003 sangramento e diminuíram após a reposição, mas mantendo-se em valores superiores aos do controle. As maiores correlações de VPS e ddown foram com IS, PAPO, PAD e ITSVE.

CONCLUSÕES: No cão, nas condições empregadas, a VPS e sua derivada ddown são indicadoras precoces de hipovolemia e guias sensíveis de reposição volêmica com SHD.

Unitermos: ANIMAL: cão; REPOSIÇÃO VOLÊMICA: solução hipertônica e hiperoncótica

\section{SUMMARY}

Paiva Filho O, Braz JRC, Silva FP, Pedro TO, Nascimento Jr P Systolic Pressure Variation as an Earlier Hypovolemia Indicator and a Guide for Volume Replacement with Hypertonic and Hyperoncotic Solution in Dogs

BACKGROUND AND OBJECTIVES: Studies have introduced a new method for preload evaluation based on systolic pressure variation analysis (SPV) during mechanical ventilation. This research aimed at evaluating whether SPV and its delta down derived (ddown) are earlier hypovolemia indicators and guides for volume replacement with hypertonic and hyperoncotic solutions.

METHODS: Twelve dogs were submitted to graded hemorrhage of $5 \%$ of their volume until reaching $20 \%$ of volume $\left(14 \mathrm{ml}^{\mathrm{kg}} \mathrm{kg}^{-1}\right)$. Before (control) and after every hemorrhage, hemodynamic, ventilatory and blood parameters were evaluated. Then, dogs were submitted to volume replacement with $7.5 \% \mathrm{NaCl}$ in $3.75 \%$ dextran 70 (SHD) $\left(4 \mathrm{ml} . \mathrm{kg}^{-1}\right)$, and the parameters were again evaluated 5 and 30 minutes after volume replacement.

RESULTS: Mean blood pressure decreased during hemorrhage and increased after SHD infusion, however without returning to baseline values. Right atrium (RAP) and pulmonary artery pressure (PAP) decreased before and increased after volume replacement reaching values similar to baseline. Pulmonary capillary wedge pressure (PCWP) decreased after the first hemorrhage and remained below baseline values even after volume replacement. Cardiac index has not changed, but increased after SHD infusion reaching values above baseline. Stroke volume index (SVI) decreased before, and increased after volume replacement reaching values above baseline. Systemic vascular and pulmonary resistance did not change during hemorrhage, but decreased after volume replacement, with SVRI remaining below baseline values and SVPRI in levels similar to baseline. Left ventricular stroke work index (LVSWI) and right ventricular stroke work index (RVSWI) decreased before and increased after SHD infusion, with RVSWI remaining above baseline values and LVSWI in levels similar to baseline. SPV and ddown progressively increased during hemorrhage and decreased after volume replacement, however remaining above baseline values. Major SPV and ddown correlations were found with SVI, PWCP, RAP, PAP and LVSWI.

CONCLUSIONS: In dogs under our experimental conditions, SPV and its derived ddown are early hypovolemia indicators and sensitive guides for volume replacement with hypertonic and hyperoncotic solutions.

Key Words: ANIMAL: dog; VOLUME REPLACEMENT: hypertonic and hyperoncotic solution 


\section{INTRODUÇÃO}

$A^{\prime}$ hipovolemia, secundária à hemorragia ou perdas líquidas para o terceiro espaço, é de difícil detecção no período operatório, porque os sinais clínicos e hemodinâmicos mais utilizados para verificar a sua ocorrência não possuem boa especificidade. Estudos experimentais introduziram um novo método para avaliação da pré-carga, baseado na análise da variação da pressão arterial sistólica durante a ventilação mecânica ${ }^{1-4}$. O aumento na pressão intratorácica durante o período inspiratório da ventilação mecânica diminui o retorno venoso e, conseqüentemente, a pressão arterial, devido à redução do volume diastólico final do ventrículo esquerdo. Assim, a variação da pressão sistólica (VPS) corresponde à diferença existente entre a pressão arterial sistólica máxima e a mínima, obtida por meio de registro durante um ciclo respiratório de ventilação controlada com volume corrente fixo. Um breve período de apnéia permite que se anule a variação da pressão arterial sistólica e se defina o seu valor de referência, a partir do qual é possível individualizar as variantes delta down (ddown) da VPS, expressa em $\mathrm{mmHg}$, correspondente à diferença entre o valor de referência e o valor mínimo da pressão arterial sistólica, e o delta up (dup), que é a diferença entre o valor máximo da pressão arterial sistólica e o valor de referência (Figura 1).

No homem, a VPS e a variante ddown mostraram-se boas indicadoras da estimação da pré-carga ${ }^{4-8}$. O estudo da VPS e de seus componentes ddown e dup demonstraram que esses atributos podem auxiliar na avaliação da reposição volêmica, de acordo com a resposta do ventrículo esquerdo ${ }^{5,6}$.

Na década de 1980, o conceito de ressuscitação com a utilização de pequenos volumes de solução hiperosmótica de cloreto de sódio a 7,5\% foi introduzido na prática clínica. Ape-

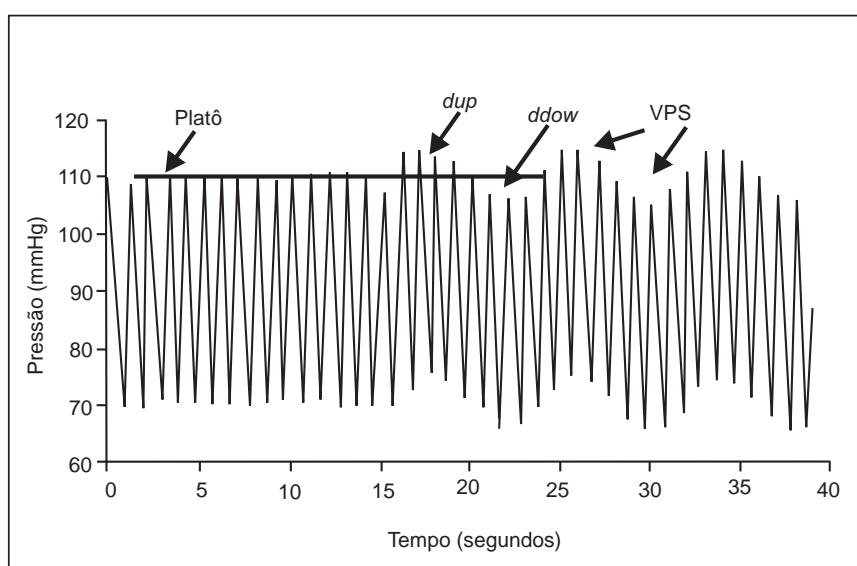

Figura 1 - A Variação da Pressão Sistólica (VPS) é calculada como a diferença entre o maior e o menor valor da pressão sistólica durante o último ciclo respiratório ou o primeiro ciclo respiratório posterior a uma fase de apnéia (platô). A VPS é separada em dois segmentos: delta up (dup) e delta down (ddown): dup é a diferença entre a maior pressão sistólica e a pressão sistólica da fase de platô (apnéia) e ddown, similarmente, é a diferença entre a pressão sistólica da fase de platô e o menor valor da pressão sistólica sar de a aplicação clínica dessa solução já ser conhecida há muitas décadas atrás, o seu uso clínico somente se popularizou a partir de 1980 , quando Velasco e col. ${ }^{9}$ relataram sobrevida importante de cães submetidos a choque hemorrágico, após receberem solução de cloreto de sódio a 7,5\%, em volume de $10 \%$ do total de sangue retirado.

A infusão da solução fisiológica hiperosmótica em pequenos volumes $\left(3\right.$ a $6 \mathrm{ml} \mathrm{kg}^{-1}$ ) aumenta rapidamente as funções cardiovascular e metabólica, por combinação de expansão do volume plasmático, com rápido deslocamento do líquido intersticial e do intracelular para o compartimento vascular, de vasodilatação sistêmica e aumento da performance miocárdica ${ }^{7,9,10}$.

Em adição ao benefício no tratamento de choque, a solução fisiológica hiperosmolar tem-se mostrado efetiva no manuseio de hipovolemia associada a procedimentos cirúrgicos maiores, como correção de aneurismas aórticos ${ }^{11}$ e revascularização do miocárdio ${ }^{12-14}$

A adição de dextran 70 à solução hiperosmolar, descrita pela primeira vez por Smith e col. (1985) ${ }^{15}$, aumenta a duração e a intensidade da expansão volêmica sem que haja perda do efeito hemodinâmico ${ }^{16}$. Enquanto a solução hiperosmolar expande o espaço vascular mobilizando água das células, inclusive das hemáceas, a adição de um colóide hiperoncótico pode, seletivamente, reter mais água no vaso. Em conseqüência, a associação das duas soluções aumenta o débito cardíaco e a taxa de sobrevida no choque hemorrágico em comparação com o uso isolado da solução hiperosmótica ${ }^{17}$. Resultados semelhantes foram obtidos com a adição de soluções de amido à solução do cloreto de sódio a $7,5 \%{ }^{18}$. Christ e col. (1997) ${ }^{19}$ sugeriram que o emprego de solução fisiológica hiperosmolar, associada à solução de dextran 70 a $6 \%$, que é hiperoncótica, abre novas perspectivas no manuseio do líquido no peri-operatório, em cirurgias eletivas e emergenciais de aneurisma de aorta abdominal. Assim, ao lado da boa estabilidade dos parâmetros hemodinâmicos, o balanço hídrico se mantém menos positivo do que durante a expansão volêmica feita apenas com solução de Ringer com lactato ou de amido, decrescendo, assim, a possibilidade de formação de edema. Além disso, parece ocorrerem efeitos microcirculatórios positivos da solução associada, que podem ser altamente benéficos em pacientes de elevado risco, como a redução do edema endotelial pós-isquêmico, da adesividade leucocitária pós-capilar e aumento da perfusão tecidual ${ }^{20,21}$.

Em revisão da literatura, não encontramos nenhuma pesquisa nacional ou internacional que correlacionasse a VPS com o emprego de solução fisiológica hiperosmolar em associação ou não com dextran em situação de choque hipovolêmico durante a anestesia.

A pesquisa experimental teve como objetivo verificar a importância da VPS, de suas derivadas ddown e dup e dos índices hemodinâmicos cardiovasculares como indicadores precoces de hipovolemia e guias de reposição volêmica, em cães submetidos à hipovolemia e à reposição volêmica com solução de cloreto de sódio a 7,5\% em associação com dex$\operatorname{tran} 70$ a $3,75 \%$. 


\section{MÉTODO}

Após aprovação pelo Comitê de Ética em Pesquisa Animal local, foram utilizados 12 cães adultos, sem raça definida, com peso entre 19 e $29 \mathrm{~kg}$. Os animais, após indução anestésica com propofol $\left(6 \mathrm{mg} \cdot \mathrm{kg}^{-1}\right)$ e fentanil $\left(5 \mu \mathrm{g} \cdot \mathrm{kg}^{-1}\right)$, por via venosa, foram posicionados na goteira de Claude Bernard e submetidos à intubação orotraqueal e ventilação controlada, com volume corrente de $20 \mathrm{ml}^{\mathrm{kg}}{ }^{-1}$ e freqüência respiratória de 12 a 15 mov. $\mathrm{min}^{-1}$, utilizando-se circuito com absorvedor de $\mathrm{CO}_{2}$, por meio do Aparelho de Anestesia Ohmeda modelo Excel 210 SE (EUA) e biomonitor AS3 da Datex Ohmeda (Finlândia). Inicialmente, realizou-se a manutenção anestésica com isoflurano a 2,7\% (duas Concentrações Alveolares Mínimas - CAM para o cão) utilizando-se vaporizador calibrado da Ohmeda (EUA) e controle das frações inspirada e expirada por meio do módulo de Análise de Gases, Halogenados e da Ventilação. Foram instalados: eletrocardiógrafo de 3 canais (derivação $D_{\|}$), sensor do termômetro no esôfago inferior, da amostra de gases ins e expirados e capnografia, junto à válvula em $Y$ do circuito respiratório e oximetria de pulso, com sensor em forma de pinça colocado na língua do animal.

Realizou-se aquecimento da superfície corporal dos animais com insuflação de ar aquecido entre 42 e $46^{\circ} \mathrm{C}$, por meio de manta específica, utilizando-se o aparelho WarmTouch da Mallinckrodt (EUA). Realizou-se dissecção e cateterismo da veia femoral direita (VFD), com cateter de polietileno PE 240, para administração da dose inicial $\left(0,6 \mathrm{mg} \cdot \mathrm{kg}^{-1}\right)$ e de manutenção $\left(10 \mu \mathrm{g} \cdot \mathrm{kg} \cdot \mathrm{min}^{-1}\right)$ do bloqueador neuromuscular brometo de rocurônio, e reposição volêmica no momento indicado, e da veia femoral esquerda (VFE), para infusão de solução de Ringer com lactato $\left(5 \mathrm{ml} \cdot \mathrm{kg}^{1} \cdot \mathrm{h}^{-1}\right)$, por meio da bomba de infusão contínua Anne, de dois canais, da Abbott (EUA).

Realizou-se dissecção e cateterismo, com cateter de polietileno PE 240, das artérias femorais direita (AFD), para sangria do animal, e esquerda (AFE), para determinação da pressão arterial média (PAM) e coleta de sangue para análise de $\mathrm{pH}$ e gases sangüíneos, no aparelho Chiron Diagnostics, modelo Rapidlab 865 (Inglaterra). Realizou-se dissecção e cateterismo da veia jugular externa direita com introdutor 8,5 F e passagem de cateter de Swan-Ganz 7F na artéria pulmonar, para medida do débito cardíaco por termodiluição e das pressões, além de coleta de sangue para análise da osmolalidade plasmática, hemoglobina, sódio e cloro, no aparelho Chiron Diagnostics, modelo Rapidlab 865 (Inglaterra). Em seguida, os animais foram submetidos à esplenectomia, por meio de laparotomia mediana.

Após o preparo, reduziu-se a CAM expirada do isoflurano para 1,4\% (uma CAM para o cão), iniciando-se o período de estabilização hemodinâmica, com duração de 10 minutos. Em seguida, registraram-se os valores das pressões arteriais média (PAM), pulmonar (PAP), de átrio direito (PAD), pulmonar ocluída (PAPO) e da VPS durante a ventilação e pausa respiratória (10 segundos). Realizou-se ainda coleta de sangue arterial e venoso misto e registro dos atributos hemodinâmicos, de ventilação, de oxigenação e de temperatura (momento Controle). Posteriormente, foram realizadas qua- tro retiradas sucessivas de $5 \%\left(3,5 \mathrm{ml}^{\mathrm{kg}}{ }^{-1}\right)$ do volume sangüíneo total ( $7 \%$ do peso corporal) do animal, totalizando $5 \%$ (momento $\mathrm{S}_{5}$ ), 10\% (momento $\mathrm{S}_{10}$ ), 15\% (momento $\mathrm{S}_{15}$ ) e $20 \%$ (momento $S_{20}$ ) da volemia do animal, sendo registrados os atributos hemodinâmicos, de ventilação, de oxigenação e de temperatura, e realizada coleta de sangue arterial e venoso misto para análise dos atributos estudados, imediatamente após cada retirada de sangue do animal. Em seguida, realizou-se reposição volêmica na VFD com solução de cloreto de sódio a $7,5 \%$ em associação com dextran 70 a 3,75\% (SHD) (4 ml. $\left.\mathrm{kg}^{-1}\right)$, em tempo total de 10 minutos, empregando-se bomba de infusão peristáltica da Arcomed (Suíça). Após 5 (momento $R V_{5}$ ) e 30 (momento $R V_{30}$ ) minutos do término da reposição volêmica, registraram-se os atributos, como descritos anteriormente, realizou-se coleta de sangue arterial e venoso misto, seguido do sacrifício do animal com excesso de anestésico (pentobarbital sódico).

Os atributos estudados foram: Respiratórios - volume corrente (VC - $\left.\mathrm{ml}^{\mathrm{kg}}{ }^{-1}\right)$, freqüência respiratória $\left(\mathrm{FR}-\mathrm{mov}_{\mathrm{min}}{ }^{-1}\right.$ ), pressão inspiratória $\left(\mathrm{PI}-\mathrm{cmH}_{2} \mathrm{O}\right)$, complacência torácica (CT - ml.cm $\left.{ }^{-1} \mathrm{H}_{2} \mathrm{O}\right)$, pressão expiratória final de $\mathrm{CO}_{2}\left(\mathrm{P}_{\mathrm{ET}} \mathrm{CO}_{2}\right.$ $\mathrm{mmHg})$ e saturação periférica da oxihemoglobina $\left(\mathrm{SpO}_{2}-\%\right)$; Hemodinâmicos - freqüência cardíaca (FC - bat.min ${ }^{-1}$ ), pressão arterial média (PAM - $\mathrm{mmHg}$ ), pressão média da artéria pulmonar (PAP - $\mathrm{mmHg}$ ), pressão média do átrio direito (PAD - $\mathrm{mmHg}$ ), pressão da artéria pulmonar ocluída (PAPO $\mathrm{mmHg}$ ), índices cardíaco (IC - L. $\mathrm{min}^{-1} \cdot \mathrm{m}^{-2}$ ), sistólico (IS $\mathrm{ml} . \mathrm{bat}^{-1} \cdot \mathrm{m}^{-2}$ ), de resistência vascular sistêmica (IRVS dina.s. $\mathrm{cm}^{-5} \cdot \mathrm{m}^{-2}$ ), de resistência vascular pulmonar (IRVP dina.s. $\left.\mathrm{cm}^{-5} \cdot \mathrm{m}^{-2}\right)$, de trabalho sistólico dos ventrículos esquerdo (ITSVE - g.min. $\mathrm{m}^{-2}$ ) e direito (ITSVD - g.min. $\mathrm{m}^{-2}$ ), variação da pressão sistólica (VPS - $\mathrm{mmHg}$ ), delta dup (dup $\mathrm{mmHg}$ ), delta down (ddown - mmHg); Sangüíneos - hemoglobina $\left(\mathrm{Hb}-\mathrm{mg}_{\mathrm{dL}}{ }^{-1}\right)$, sódio plasmático $\left(\mathrm{Na}^{+}-\mathrm{mMol}^{-1} \mathrm{~L}^{-1}\right)$, cloro plasmático $\left(\mathrm{Cl}^{-}-\mathrm{mMol} . \mathrm{L}^{-1}\right)$, osmolalidade plasmática (Oplasm - mOsm.kg ${ }^{-1} \mathrm{H}_{2} \mathrm{O}$ ), $\mathrm{pH}$ arterial ( $\mathrm{pHa}$ ) e pressão parcial de dióxido de carbono arterial $\left(\mathrm{PaCO}_{2}-\mathrm{mm} \mathrm{Hg}\right)$; Temperatura - esofagiana $\left(\mathrm{T}_{\mathrm{Esof}}-{ }^{\circ} \mathrm{C}\right)$. A SHD foi preparada com a associação da solução de cloreto de sódio a $20 \%(37,5 \mathrm{ml})$ à solução de dextran 70 a $6 \%$ em glicose a $5 \%$ (62,5 ml). Dessa forma a solução final ( $100 \mathrm{ml}$ ) continha $\mathrm{NaCl}$ a $7,5 \%$ em solução de dextran 70 a 3,75\%.

Não existe um sistema de monitorização que realize o cálculo automático da VPS. Para isto é necessário que se registre a curva da pressão arterial durante alguns ciclos respiratórios e durante uma fase de apnéia, utilizando-se pequena velocidade do papel de impressão (Figura 1). Na determinação dos atributos hemodinâmicos que foram obtidos indiretamente, utilizou-se software do biomonitor AS3, com a introdução dos valores dos atributos necessários.

\section{Análise Estatística}

Como a distribuição dos atributos foi não-paramétrica, aplicou-se inicialmente o teste de Friedman. Os contrastes entre as medianas do grupo foram verificados pelo método de Student-Newman-Keuls. Nessa situação, os resultados são 
apresentados por meio de tabelas ou figuras, com indicação dos valores da mediana, primeiro (25\%) e terceiro (75\%) quartis dos atributos estudados no grupo. Foram feitas também regressões lineares de Pearson para determinar a correlação entre VPS, ddown e dup com os demais atributos hemodinâmicos estudados. Níveis de significância menores do que 0,05 foram considerados significantes.

\section{RESULTADOS}

Os valores antropométricos e a distribuição de machos e fêmeas no grupo estudado são vistos na tabela I.

Tabela I - Valores Antropométricos e Distribuição do Sexo no Grupo Estudado

\begin{tabular}{lccc}
\hline Peso $(\mathrm{kg}){ }^{*}$ & Comprimento $(\mathrm{cm})^{*}$ & Superfície corporal $^{*}$ & Sexo $(\mathrm{M} / \mathrm{F})$ \\
\hline $23,7 \pm 3,0$ & $107,4 \pm 6,6$ & $0,81 \pm 0,07$ & $11 / 1$ \\
\hline
\end{tabular}

* Valores expressos pela Média \pm DP
Os resultados dos atributos ventilatórios estão apresentados na tabela II. Não houve alteração significante dos atributos ventilatórios estudados, com exceção da $\mathrm{P}_{\mathrm{ET}} \mathrm{CO}_{2}$ que aumentou significativamente em relação ao controle, nos momentos finais do sangramento e, ainda mais, após a reposição volêmica.

Os resultados da hemodinâmica cardiovascular são vistos nas tabelas III e IV e nas figuras 2 e 3 . Os valores da FC, embora se elevassem, não se alteraram significativamente durante os momentos de sangramento e após a reposição volêmica. Os valores da pressão arterial média diminuíram significativamente durante os momentos de sangramento e aumentaram significativamente após a reposição volêmica, mas sem atingirem os valores do controle. Os valores da pressão média da artéria pulmonar diminuíram significativamente e progressivamente durante os momentos de sangramento e aumentaram significativamente nos momentos seguintes à reposição volêmica para valores semelhantes aos do controle. Os valores da pressão média do átrio direito diminuíram significativamente durante o sangramento e aumentaram significativamente nos momentos seguintes à re-

Tabela II - Valores da Mediana, 1e e ${ }^{\circ}$ Quartis do Volume Corrente (VC), Freqüência Respiratória (FR), Pressão Inspiratória $\left(\mathrm{P}_{1}\right)$, Complacência Torácica $(C T)$, Pressão Expiratória Final de Dióxido de Carbono $\left(\mathrm{P}_{\mathrm{ET}} \mathrm{CO}_{2}\right)$ e Saturação Periférica de Oxihemoglobina $\left(\mathrm{SpO}_{2}\right)$ Obtidos no Grupo Estudado

\begin{tabular}{|c|c|c|c|c|c|c|}
\hline \multirow[t]{2}{*}{ Momentos } & \multicolumn{6}{|c|}{ Atributos } \\
\hline & VC $\left(\mathrm{ml} . \mathrm{kg}^{-1}\right)$ & $\mathrm{FR}\left(\mathrm{mov}^{\mathrm{min}} \mathrm{m}^{-1}\right)$ & $\mathrm{PI}\left(\mathrm{cmH}_{2} \mathrm{O}\right)$ & CT $\left(\mathrm{ml} . \mathrm{cm}^{-1} \mathrm{H}_{2} \mathrm{O}\right)$ & $\mathrm{P}_{\mathrm{ET}} \mathrm{CO}_{2}(\mathrm{mmHg})$ & $\mathrm{SpO}_{2}(\%)$ \\
\hline Controle & $19,5[18,8 ; 20,4] \mathrm{a}^{(1)}$ & $15,5[10,5 ; 18,0] a$ & $15,0[14,0 ; 17,0] a$ & $34,0[32,0 ; 41,5] \mathrm{a}$ & $34,0[33,5 ; 35,5] \mathrm{a}$ & $99,0[97,0 ; 100,0] \mathrm{a}$ \\
\hline $\mathrm{S}_{5}$ & $19,5[18,6 ; 20,2] a$ & $15,5[11,5 ; 18,0] a$ & $15,0[14,5 ; 17,0] a$ & $34,0[31,5 ; 42,0] a$ & $35,0[33,5 ; 36,0] a$ & $99,0[98,0 ; 100,0] a$ \\
\hline $\mathrm{S}_{10}$ & $19,5[18,6 ; 20,2] a$ & $15,5[11,5 ; 18,0] a$ & $15,0[14,5 ; 16,0] a$ & $33,5[31,0 ; 40,5] a$ & $36,0[34,0 ; 36,0] a$ & $99,0[98,5 ; 100,0] a$ \\
\hline $\mathrm{S}_{15}$ & $19,5[18,6 ; 20,2] a$ & $15,5[11,5 ; 18,0] a$ & $15,0[14,5 ; 17,0] \mathrm{a}$ & $34,0[30,5 ; 41,5] a$ & $36,0[33,5 ; 38,0] \mathrm{b}$ & $99,0[98,5 ; 100,0] a$ \\
\hline $\mathrm{S}_{20}$ & $19,1[18,4 ; 20,0] a$ & $15,5[11,5 ; 18,0] a$ & $15,5[14,5 ; 17,5] a$ & $35,0[30,5 ; 42,0] a$ & $38,0[35,5 ; 38,0] c$ & $99,0[98,0 ; 100,0] a$ \\
\hline $\mathrm{RV}_{5}$ & $19,2[18,4 ; 19,6] a$ & $15,5[11,5 ; 18,0] a$ & $15,5[14,0 ; 17,0] \mathrm{a}$ & $37,0[31,0 ; 41,5] \mathrm{a}$ & $42,0[38,5 ; 44,5] \mathrm{d}$ & $99,0[97,5 ; 99,0] a$ \\
\hline $\mathrm{RV}_{30}$ & $19,1[18,6 ; 20,7] a$ & $15,5[11,5 ; 18,0] a$ & $16,0[14,0 ; 16,5] a$ & $36,5[30,5 ; 42,0] a$ & $40,0[38,0 ; 43,5] d$ & $99,0[98,0 ; 99,0] a$ \\
\hline
\end{tabular}

(1) Momentos representados por medianas seguidas de letras minúsculas diferentes no grupo diferem significativamente $(p<0,05)$

Tabela III - Valores da Mediana, 1e e $3^{\circ}$ Quartis da Freqüência Cardíaca (FC), Pressão Arterial Média (PAM), Pressão do Átrio Direito (PAD), Pressão da Artéria Pulmonar (PAP), Pressão da Artéria Pulmonar Ocluída (PAPO) e Índice Cardíaco (IC) Obtidos no Grupo Estudado

\begin{tabular}{|c|c|c|c|c|c|c|}
\hline \multirow[t]{2}{*}{ Momentos } & \multicolumn{6}{|c|}{ Atributos } \\
\hline & $\mathrm{FC}\left(\right.$ bat. $\left.\mathrm{min}^{-1}\right)$ & PAM $(\mathrm{mmHg})$ & PAD $(\mathrm{mmHg})$ & $\mathrm{PAP}(\mathrm{mmHg})$ & PAPO $(\mathrm{mmHg})$ & IC $\left(\right.$ L. $\left.\mathrm{min}^{-1} \cdot \mathrm{m}^{-2}\right)$ \\
\hline Controle & $128,0[113 ; 145] a^{(1)}$ & $90,5[85,5 ; 99,0] c$ & $3,0[3,0 ; 3,5] \mathrm{c}$ & $12,0[11,5 ; 15,0] d$ & $5,0[4,0 ; 7,0] \mathrm{b}$ & $3,50[2,75 ; 4,30] \mathrm{a}$ \\
\hline $\mathrm{S}_{5}$ & $134,0[111 ; 147] \mathrm{a}$ & $80,5[77,0 ; 96,0] \mathrm{b}$ & $2,0[2,0 ; 3,0] \mathrm{b}$ & $12,0[10,5 ; 14,0] d$ & $3,5[2,5 ; 6,0] a$ & $3,70[2,75 ; 3,80] a$ \\
\hline $\mathrm{S}_{10}$ & $135,0[119 ; 156] a$ & $79,0[72,0 ; 84,0] \mathrm{b}$ & $2,0[1,5 ; 3,0] a$ & $11,5[10,0 ; 14,0] \mathrm{c}$ & $3,5[2,5 ; 4,5] a$ & $3,35[2,85 ; 3,80] a$ \\
\hline $\mathrm{S}_{15}$ & $141,0[123 ; 159] a$ & $73,0[71,0 ; 79,0] \mathrm{b}$ & $2,0[1,0 ; 2,5] \mathrm{a}$ & $10,0[9,0 ; 12,5] \mathrm{b}$ & $2,0[2,0 ; 3,0] a$ & $3,20[2,75 ; 3,65] a$ \\
\hline $\mathrm{S}_{20}$ & $146,0[121 ; 166] a$ & $74,0[67,0 ; 80,5] a$ & $2,0[1,0 ; 2,5] a$ & $9,5[9,0 ; 11,5] \mathrm{a}$ & $2,0[1,5 ; 3,0] a$ & $3,45[2,90 ; 3,80] a$ \\
\hline $\mathrm{RV}_{5}$ & $143,0[128 ; 162] a$ & $79,0[75,5 ; 84,5] \mathrm{b}$ & $3,0[2,0 ; 3,0] d$ & $13,011,5 ; 14,0] \mathrm{d}$ & $3,0[2,0 ; 4,0] a$ & $5,05[4,40 ; 5,75] \mathrm{c}$ \\
\hline $\mathrm{RV}_{30}$ & $142,0[132 ; 163] a$ & $83,5[71,5 ; 93,0] \mathrm{b}$ & $2,0[1,5 ; 2,0] \mathrm{c}$ & $12,5[11,5 ; 14,5] d$ & $3,0[2,0 ; 4,5] a$ & $4,70[4,20 ; 5,30] \mathrm{b}$ \\
\hline
\end{tabular}

(1) Momentos representados por medianas seguidas de letras minúsculas diferentes no grupo diferem significativamente $(p<0,05)$ 
Tabela IV - Valores da Mediana, 1ำ e 3ํQuartis do Índice Sistólico (IS), Índice de Resistência Vascular Sistêmica (IRVS), Índice de Resistência Vascular Pulmonar (IRVP), Índice do Trabalho Sistólico do Ventrículo Esquerdo (ITSVE), Índice do Trabalho Sistólico do Ventrículo Direito (ITSVD) e Delta up (dup) Obtidos no Grupo Estudado

\begin{tabular}{|c|c|c|c|c|c|c|}
\hline \multirow[t]{2}{*}{ Momentos } & \multicolumn{6}{|c|}{ Atributos } \\
\hline & IS $\left(\mathrm{ml}^{\mathrm{b}} \mathrm{bat}^{-1} \cdot \mathrm{m}^{-2}\right)$ & IRVS (dina.s. $\mathrm{cm}^{-5} \cdot \mathrm{m}^{-2}$ ) & IRVP (dina.s. $\mathrm{cm}^{-5} \cdot \mathrm{m}^{-2}$ ) & ITSVE (g.min. $\mathrm{m}^{-2}$ ) & ITVSD (g.min. $\mathrm{m}^{-2}$ ) & Dup (mmHg) \\
\hline Controle & $28,0[24,5 ; 32,0] \mathrm{d}^{(1)}$ & 2133[1658;2679]b & $156[112 ; 244] \mathrm{b}$ & $35,3[29,1 ; 39,8] d$ & $3,20[2,40 ; 4,70] \mathrm{c}$ & $2,0[0,0 ; 2,5] a$ \\
\hline $\mathrm{S}_{5}$ & $27,0[22,5 ; 29,5] \mathrm{c}$ & $1944[1656 ; 2489] \mathrm{b}$ & $147[131 ; 271] \mathrm{b}$ & $29,0[26,2 ; 35,6] \mathrm{c}$ & $3,20[2,60 ; 3,95] \mathrm{c}$ & $0,6[0,0 ; 1,5] a$ \\
\hline $\mathrm{S}_{10}$ & $25,0[22,0 ; 28,0] \mathrm{b}$ & $1769[1675 ; 2184] \mathrm{b}$ & $169[137 ; 207] \mathrm{b}$ & $26,0[22,9 ; 29,8] \mathrm{b}$ & $3,05[2,05 ; 3,80] \mathrm{b}$ & $0,0[0,0 ; 1,4] \mathrm{a}$ \\
\hline $\mathrm{S}_{15}$ & $24,5[19,5 ; 29,5] a$ & $1776[1552 ; 2057] \mathrm{b}$ & $190[144 ; 263] b$ & $27,1[19,6 ; 30,8] \mathrm{b}$ & $3,10[1,95 ; 3,60] \mathrm{b}$ & $0,0[0,0 ; 1,9] \mathrm{a}$ \\
\hline $\mathrm{S}_{20}$ & $23,0[20,5 ; 27,0] a$ & $1633[1505 ; 2360] \mathrm{b}$ & $188[124 ; 273] \mathrm{b}$ & $23,5[18,2 ; 26,9] a$ & $2,70[2,00 ; 3,15] a$ & $0,0[0,0 ; 0,6] \mathrm{a}$ \\
\hline $\mathrm{RV}_{5}$ & $37,0[28,0 ; 40,5] \mathrm{f}$ & $1259[1179 ; 1633] a$ & $140[119 ; 195] a$ & $35,0[30,5 ; 44,5] \mathrm{e}$ & $4,80[3,80 ; 5,70] d$ & $0,0[0,0 ; 0,1] a$ \\
\hline $\mathrm{RV}_{30}$ & $31,5[26,5 ; 38,0] \mathrm{e}$ & $1369[1240 ; 1783] a$ & $149[132 ; 223] b$ & $32,7[29,6 ; 42,4] d$ & $4,40[3,55 ; 5,65] d$ & $0,6[0,0 ; 1,9] a$ \\
\hline
\end{tabular}

(1) Momentos representados por medianas seguidas de letras minúsculas diferentes no grupo diferem significativamente $(p<0,05)$

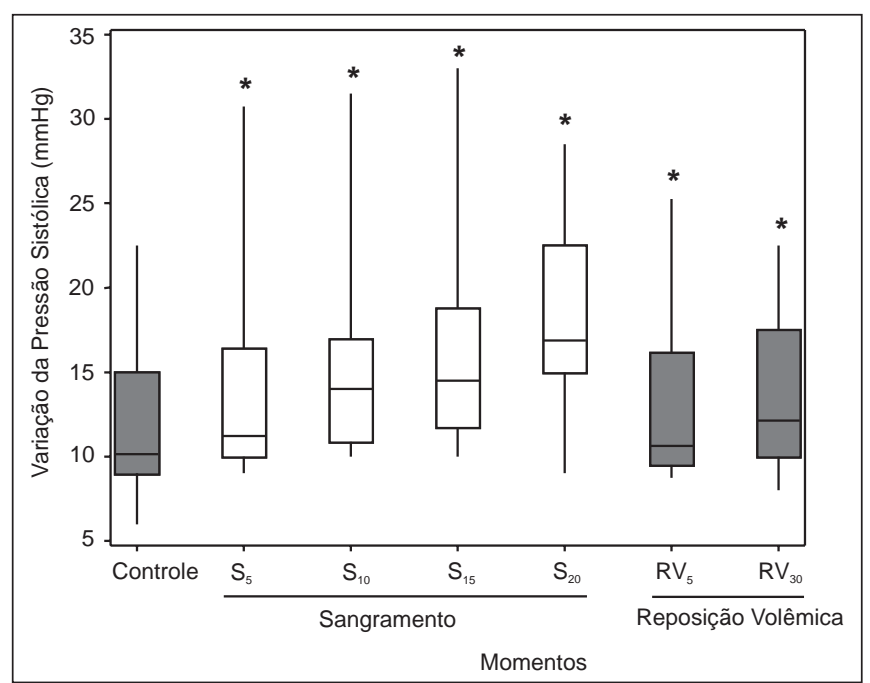

Figura 2 - Box Plot Apresentando os Valores Mínimos, do 1ํQuartil, da Mediana, do 3ํㅡuartil e Máximo da Variação da Pressão Sistólica nos Momentos Estudados

${ }^{*} \mathrm{p}<0,05: \mathrm{C}<\left(\mathrm{RV}_{5}=\mathrm{RV}_{30}=\mathrm{S}_{5}\right)<\mathrm{S}_{10}<\left(\mathrm{S}_{15}=\mathrm{S}_{20}\right)$

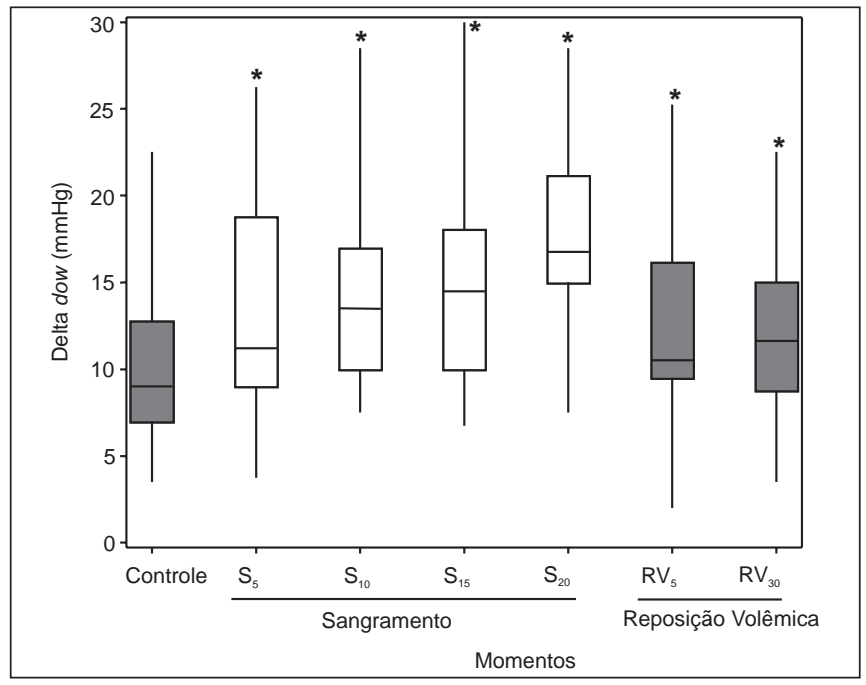

Figura 3 - Box Plot Apresentando os Valores Mínimos, do 1ํQuartil, da Mediana, do 3ํㅡㄴ Quartil e Máximo do ddown nos Momentos Estudados

${ }^{*} \mathrm{p}<0,05: \mathrm{C}<\left(\mathrm{S}_{5}=\mathrm{RV}_{5}=\mathrm{RV}_{30}\right)<\mathrm{S}_{10}<\mathrm{S}_{15}<\mathrm{S}_{20}$

Revista Brasileira de Anestesiologia

Vol. 53, № 3, Maio - Junho, 2003 posição volêmica para valores inicialmente superiores aos do controlee, após 30 minutos, semelhantes aos do controle. Os valores da pressão da artéria pulmonar ocluída diminuíram significativamente após o primeiro período de sangramento, mantendo-se, a seguir, nos demais momentos do sangramento e da reposição volêmica, em valores inferiores aos do controle. Os valores do índice cardíaco não se alteraram significativamente durante os momentos de sangramento, mas aumentaram significativamente nos momentos seguintes à reposição volêmica, para valores superiores aos do controle.

Os valores de IS diminuíram significativamente e progressivamente durante o sangramento e aumentaram significativamente após a reposição volêmica, em níveis superiores aos do controle. Os valores do IRVS e IRVP não se alteraram significativamente durante o sangramento, mas diminuíram significativamente após a reposição volêmica, sendo que no momento final do estudo $\left(R V_{30}\right)$, IRVS manteve-se em níveis inferiores aos do controle e a IRVP manteve-se em níveis semelhantes aos do controle. Já ITSVE e ITSVD diminuíram significativamente durante o sangramento, mas aumentaram significativamente após a reposição volêmica, sendo que em $\mathrm{RV}_{30}$, o ITSVD manteve-se em níveis superiores aos do controle e o ITSVE manteve-se em níveis semelhantes aos do controle.

Os valores da VPS e de ddown aumentaram significativamente e progressivamente ao longo do sangramento e diminuíram significativamente após a reposição volêmica, mas ainda se mantendo em valores superiores aos do controle (Figuras 2 e 3). Já os valores de dup não se alteraram significativamente.

Os níveis de sódio e cloro plasmáticos aumentaram significativamente após a reposição volêmica (Figura 4 e Tabela V), acarretando aumento significante da osmolalidade plasmática (Figura 5). Os níveis da Hb, que já haviam diminuído progressivamente após cada sangramento realizado, diminuíram ainda mais após a reposição volêmica (Tabela V). $\mathrm{O}$ pHa diminuiu significativamente com o sangramento e após a reposição volêmica, enquanto a $\mathrm{PaCO}_{2}$ aumentou significativamente após a reposição volêmica (Tabela $\mathrm{V}$ ). Já a $\mathrm{T}_{\text {Esof }}$ não se alterou significativamente (Tabela V). 


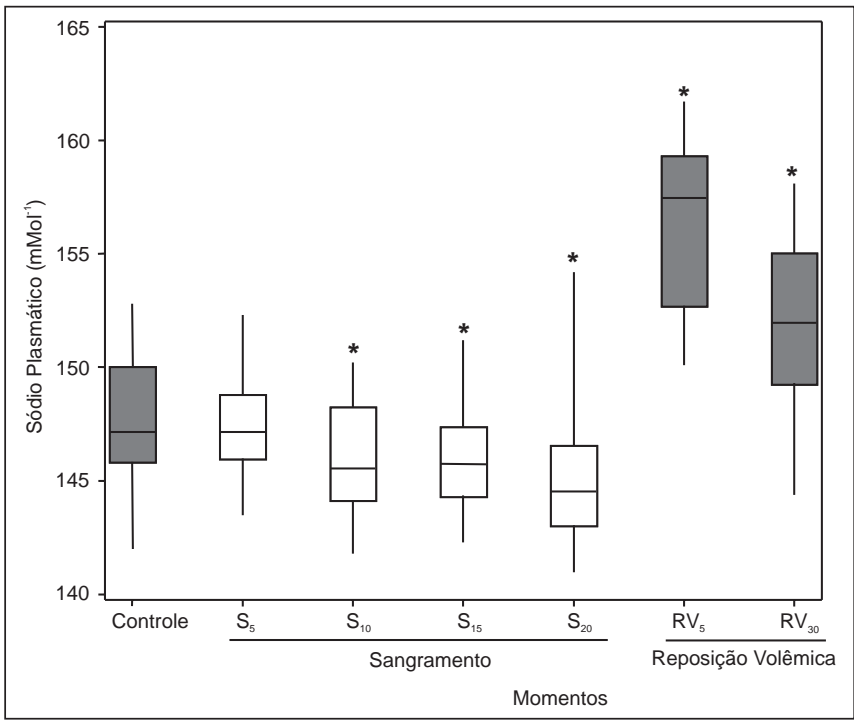

Figura 4 - Box Plot Apresentando os Valores Mínimo, do 1 Quartil, da Mediana, do 3ํㅡuartil e Máximo do Sódio Plasmático nos Momentos Estudados

${ }^{*} \mathrm{p}<0,05:\left(\mathrm{S}_{10}=\mathrm{S}_{15}=\mathrm{S}_{30}\right)<\left(\mathrm{C}=\mathrm{S}_{5}\right)<\mathrm{RV}_{30}<\mathrm{RV}_{5}$

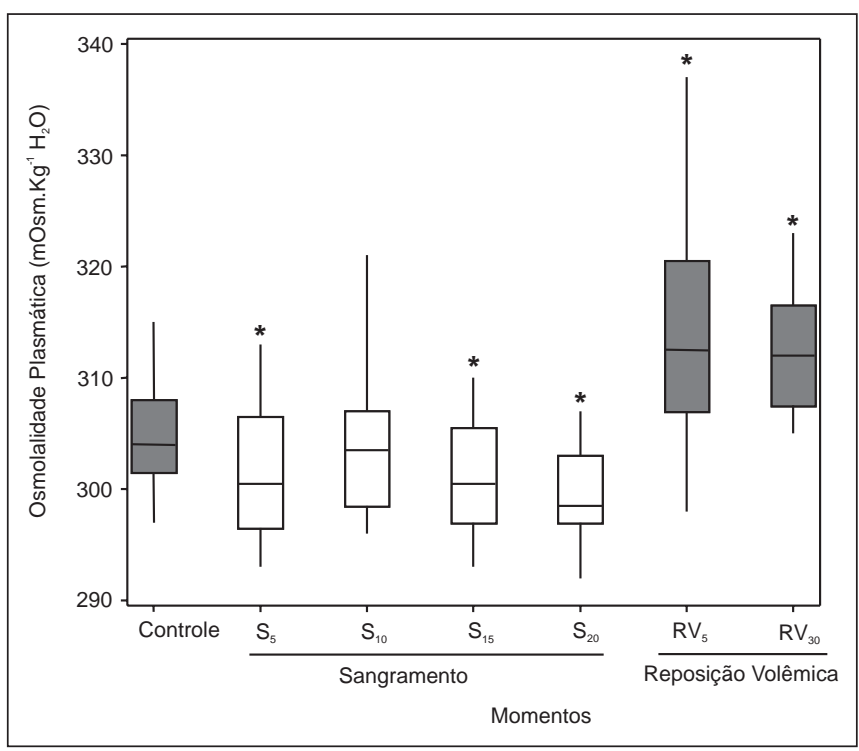

Figura 5 - Box Plot Apresentando os Valores Mínimos, do $1^{\circ}$ Quartil, da Mediana, do 3을 Quartil e Máximo da Osmolalidade Plasmática nos Momentos Estudados

${ }^{*} \mathrm{p}<0,05: \mathrm{S}_{20}<\left(\mathrm{S}_{5}=\mathrm{S}_{15}\right)<\left(\mathrm{C}=\mathrm{S}_{10}\right)<\left(\mathrm{RV}_{5}=\mathrm{RV}_{30}\right)$
O coeficiente de correlação de Pearson da variação da pressão sistólica (VPS) e ddown com os demais atributos hemodinâmicos ao longo do experimento, com indicação dos valores de r e p estão apresentados na tabela VI.

Tabela VI - Coeficiente de Correlação de Pearson da Variação da Pressão Sistólica (VPS), ddown e dup com os Demais Atributos Hemodinâmicos ao Longo do Experimento $(n=84)$, com Indicação dos Valores de $r$ e $p$

\begin{tabular}{llll}
\hline Atributos & \multicolumn{1}{c}{ VPS } & \multicolumn{1}{c}{ ddown } & \multicolumn{1}{c}{ dup } \\
\hline Pressão arterial & $\mathrm{r}=-0,23751$ & $\mathrm{r}=-0,20551$ & $\mathrm{r}=-0,07062$ \\
média & $\mathrm{p}=0,0296$ & $\mathrm{p}=0,0613$ & $\mathrm{p}=0,5233$ \\
& $\mathrm{r}=-0,43758$ & $\mathrm{r}=-0,36921$ & $\mathrm{r}=-0,21172$ \\
Índice cardíaco & $\mathrm{p}=0,0001$ & $\mathrm{p}=0,0005$ & $\mathrm{p}=0,0532$ \\
Pressão da artéria & $\mathrm{r}=-0,22916$ & $\mathrm{r}=-0,25773$ & $\mathrm{r}=0,15129$ \\
pulmonar & $\mathrm{p}=0,0360$ & $\mathrm{p}=0,0179$ & $\mathrm{p}=0,1695$ \\
Pressão de átrio & $\mathrm{r}=-0,44495$ & $\mathrm{r}=-0,53651$ & $\mathrm{r}=0,41326$ \\
direito & $\mathrm{p}=0,0001$ & $\mathrm{p}=0,0001$ & $\mathrm{p}=0,0001$ \\
Pressão da artéria & $\mathrm{r}=-0,47252$ & $\mathrm{r}=-0,57140$ & $\mathrm{r}=0,43389$ \\
pulmonar ocluída & $\mathrm{p}=0,0001$ & $\mathrm{p}=0,0001$ & $\mathrm{p}=0,0001$ \\
& $\mathrm{r}=-0,59646$ & $\mathrm{r}=-0,56753$ & $\mathrm{r}=-0,03708$ \\
Índice sistólico & $\mathrm{p}=0,0001$ & $\mathrm{p}=0,0001$ & $\mathrm{p}=0,7377$ \\
& $\mathrm{r}=-0,40938$ & $\mathrm{r}=-0,36932$ & $\mathrm{r}=-0,09989$ \\
Índice de trabalho & $\mathrm{p}=0,0001$ & $\mathrm{p}=0,0001$ & $\mathrm{p}=0,3660$ \\
sistólico VD & $\mathrm{r}=-0,54229$ & $\mathrm{r}=-0,49679$ & $\mathrm{r}=-0,09982$ \\
Índice de trabalho & $\mathrm{p}=0,0001$ & $\mathrm{p}=0,0001$ & $\mathrm{p}=0,3663$ \\
sistólico VE & $\mathrm{r}=0,33201$ & $\mathrm{r}=0,27765$ & $\mathrm{r}=0,18839$ \\
Índice de resistência & $\mathrm{p}=0,0020$ & $\mathrm{p}=0,0001$ & $\mathrm{p}=0,0861$ \\
vascular sistêmica & & $\mathrm{r}=0,96708$ & $\mathrm{r}=-0,00327$ \\
VPS & - & $\mathrm{p}=0,0001$ & $\mathrm{p}=0,9765$ \\
& & - & $\mathrm{r}=-0,24955$ \\
ddown & $\mathrm{r}=0,96708$ & $\mathrm{p}=0,0221$ \\
\hline
\end{tabular}

A correlação entre VPS e ddown foi elevada. A correlação desses atributos com os demais atributos hemodinâmicos foram, em sua maioria, significantes. As maiores correlações de VPS foram, em ordem decrescente, com: ddown, IS, ITSVE, PAPO, PAP, IC e ITSVD, enquanto as de ddown foram, em ordem decrescente, com: VPS, PAPO, IS, PAD e ITSVE. Já dup teve as maiores correlações com a PAPO e a PAD.

Na análise da correlação de Pearson em cada momento, isoladamente, verificou-se, em todos os momentos, correlação significante e elevada ( $r$ sempre acima de 0,90 ) entre VPS e ddown. No controle, a única correlação significante que ocorreu foi de ddown com a PAPO. Nos momentos de sangramento, VPS e ddown tiveram correlação significante com IS,

Tabela V - Valores da Mediana, 1ํ e 3ํQuartis da Hemoglobina (Hb), Cloro (Cl) Plasmático, pH arterial (pHa), Pressão Expiratória Final do Dióxido de Carbono Arterial $\left(\mathrm{PaCO}_{2}\right)$ e Temperatura Esofagiana ( $\left.\mathrm{T}_{\text {Esof }}\right)$ Obtidos no Grupo Estudado

\begin{tabular}{|c|c|c|c|c|c|}
\hline \multirow[t]{2}{*}{ Momentos } & \multicolumn{5}{|c|}{ Atributos } \\
\hline & $\mathrm{Hb}\left(\mathrm{mg} \cdot \mathrm{dl}^{-1}\right)$ & $\mathrm{Cl}-\left(\mathrm{mOsm} \cdot \mathrm{L}^{-1}\right)$ & $\mathrm{pHa}$ & $\mathrm{PaCO}_{2}(\mathrm{mmHg})$ & Tesof $\left({ }^{\circ} \mathrm{C}\right)$ \\
\hline Controle & $13,0[12,3 ; 14,2] \mathrm{g}^{(1)}$ & $110,5[109,0 ; 112,0] \mathrm{a}$ & $7,38[7,33 ; 7,45] \mathrm{d}$ & $36,2[34,8 ; 38,6] \mathrm{a}$ & $38,3[37,7 ; 39,3] \mathrm{a}$ \\
\hline $\mathrm{S}_{5}$ & $13,3[12,5 ; 13,9] f$ & $110,0[108,5 ; 112,0] a$ & $7,38[7,31 ; 7,44] \mathrm{c}$ & $38,6[35,7 ; 39,9] a$ & $38,3[37,7 ; 39,1] \mathrm{a}$ \\
\hline$S_{10}$ & $12,3[11,9 ; 13,6] \mathrm{e}$ & $110,0[107,5 ; 112,0] a$ & $7,38[7,32 ; 7,41] \mathrm{c}$ & $37,5[36,5 ; 42,5] \mathrm{a}$ & $38,2[37,7 ; 39,1] \mathrm{a}$ \\
\hline$S_{15}$ & $12,5[11,9 ; 13,5] d$ & $110,5[108,0 ; 112,0] a$ & $7,36[7,30 ; 7,42] \mathrm{c}$ & $40,1[36,5 ; 43,5] \mathrm{a}$ & $38,2[37,7 ; 39,0] a$ \\
\hline $\mathrm{S}_{20}$ & $11,9[11,1 ; 13,2] \mathrm{c}$ & $110,0[109,0 ; 112,5] \mathrm{a}$ & $7,34[7,29 ; 7,40] \mathrm{b}$ & $37,8[35,4 ; 42,3] \mathrm{a}$ & $38,3[37,8 ; 39,0] \mathrm{a}$ \\
\hline $\mathrm{RV}_{30}$ & $11,1[10,6 ; 11,3] a$ & $119,5[117,0 ; 122,5] \mathrm{b}$ & $7,30[7,25 ; 7,38] \mathrm{a}$ & $44,5[39,8 ; 48,1] \mathrm{b}$ & $38,3[37,8 ; 38,7] \mathrm{a}$ \\
\hline
\end{tabular}

(1) Momentos representados por medianas seguidas de letras minúsculas diferentes no grupo diferem significativamente $(p<0,05)$ 
ITSVE e PAPO, além do IC em $\mathrm{RV}_{30}$. Após a reposição com SHD, houve correlação significante da VPS e ddown com ISe PAPO, enquanto ddown, isoladamente, apresentou interação significante também com a PAD e a ITSVE.

\section{DISCUSSÃO}

Optou-se pelo cão de porte médio a grande por apresentar padrões hemodinâmicos compatíveis com os do homem e tamanho apropriado para suportar a preparação cirúrgica empregada na pesquisa e facilitar a monitorização hemodinâmica, por meio da instalação de cateter na artéria pulmonar. A manutenção do volume corrente, pressão inspiratória e complacência em valores uniformes ao longo do experimento foi importante, pois VPS, ddown e dup sofrem influência desses atributos. Ovolume corrente utilizado foi semelhante ao do cão em vigília $\left(20 \mathrm{ml}^{\mathrm{kg}}{ }^{-1}\right)^{22}$. Perel e col (1987) ${ }^{1}$ utilizaram volume corrente de $12 \mathrm{ml}^{.} \mathrm{kg}^{-1}$ e veste inflável em torno do tórax do cão para manter a complacência torácica semeIhante à do homem. Nesse e em nosso estudo, quando não se utilizou veste inflável no cão, a complacência torácica foi semelhante a do homem. Os diferentes volumes correntes empregados nas duas pesquisas podem explicar os resultados obtidos.

O volume de sangue circulante do cão adulto e saudável é de $7 \%$ do peso corporal ${ }^{1}$ e o volume sangüíneo retirado de cada animal foi de $14 \mathrm{ml} . \mathrm{kg}^{-1}$ determinando hipovolemia de graus I a II (Committe on Trauma) ${ }^{23}$. O fato de a retirada de sangue ter sido realizada em quatro períodos, com intervalo de 15 minutos entre eles, certamente evitou que houvesse alterações cardiocirculatórias mais importantes do que as que ocorreram, pois permitiu que o animal compensasse, ao menos parcialmente, a perda da volemia.

Tanto o volume plasmático como o de hemáceas são parcialmente restaurados na fase inicial do choque hemorrágico ( $g r a u l)$, mas a intensidade desses ajustes varia entre os indivíduos e as espécies.

Com a ativação do sistema simpático ocorre constrição dos vasos de capacitância, que provoca grande mobilização central do sangue localizado nas grandes e pequenas veias, com aumento na pré-carga. Nessa fase também começa a ocorrer vasoconstrição arterial, com restrição de fluxo aos territórios cutâneo, muscular esquelético, renal e esplâncnico, enquanto no cérebro e miocárdio ocorre vasodilatação mediada localmente.

A restauração do volume plasmático resulta da vasoconstrição seletiva pré-capilar, que reduz a pressão hidrostática do sistema microcirculatório. Como resultado, a pressão oncótica passa a predominar, com absorção de fluido intersticial. Esse fenômeno é conhecido como autotransfusão interna ou shift transcapilar, que compensa as perdas plasmáticas e tende a provocar hemodiluição ${ }^{24}$.

A restauração do volume de hemáceas circulantes é também dependente da ativação simpática, com esplenoconstrição, que é muito eficiente no cão, por causa do grande volume do baço, conseguindo transferir para a circulação as reservas de sangue, com elevado hematócrito, acumuladas no parênquima esplâncnico.

Aesplenectomia, para melhor controle da volemia durante os períodos de sangramento causou hipovolemia relativa nos animais, considerando-se que a reposição volêmica nesse período com Ringer com lactato foi pequena $\left(5 \mathrm{ml} \cdot \mathrm{kg}^{-1} \cdot \mathrm{h}^{-1}\right) . \mathrm{O}$ fato da VPS em muitos cães ser constituída apenas por ddown no momento controle reforça essa idéia. Os demais atributos hemodinâmicos estudados estavam dentro da faixa de normalidade para o cão sob anestesia ${ }^{22}$, o que reforça, ainda mais a importância da VPS e de ddown como indicadores precoces de hipovolemia. Durante o sangramento e situações de hipovolemia, como se verificou na pesquisa, o ddown pode aumentar muito tornando-se responsável por praticamente toda a VPS ${ }^{1,6}$. Os valores normais de ddown são de 5 a $6 \mathrm{mmHg}$, do dup de 2 a $4 \mathrm{mmHg}$ e da VPS de 8 a 10 $\mathrm{mmHg}{ }^{5,6}$.

A detecção da hipovolemia insidiosa no per-operatório é difícil e alguns atributos, como a FC e a PAM, utilizados clinicamente, nem sempre são bons indicadores de alteração volêmica, como verificamos na presente pesquisa. Mesmo a PAPO, considerada importante ${ }^{12}$, apresenta limitações como indicadora do volume diastólico final do ventrículo esquerdo ${ }^{5,25,26}$.

Na presente pesquisa, durante os momentos de sangramento, ddown foi o atributo que apresentou melhor correlação com cada nível de sangramento, junto com a hemoglobina. Os atributos que também apresentaram boa correlação em cada fase do sangramento foram: VPS, PAP, IS e ITSVD. Alguns atributos não apresentaram nenhuma correlação com os níveis de sangramento, como FC, IC, IRVS, IRVP e dup.

Assim, tanto a VPS como ddown têm se mostrado indicadores sensíveis de hipovolemia no cão ${ }^{1-4}$ e no homem ${ }^{5-7,27}$. Durante a fase inspiratória da ventilação, o aumento das pressões intratorácica e intraluminal do átrio direito aumentam a resistência ao retorno venoso, diminuindo a pré-carga do átrio direito e o volume de ejeção do ventrículo direito. No paciente hipovolêmico, esta redução é ainda mais importante. Após alguns batimentos cardíacos, a diminuição do volume ejetado pelo ventrículo direito repercutirá no ventrículo esquerdo. A diminuição do volume de ejeção do ventrículo esquerdo ocorre em proporções idênticas à diminuição do volume de ejeção do ventrículo direito, e provoca diminuição da pressão arterial sistólica. A diminuição da pressão arterial sistólica, que ocorre na fase expiratória, poderá ser interrompida pela insuflação do ciclo respiratório seguinte, caso a freqüência respiratória seja elevada, superior a 20 ciclos. min $^{-1}$ 28. A influência da ventilação controlada sobre o retorno venoso é limitada quando ocorre diminuição de complacência pulmonar ou aumento da complacência torácica ${ }^{1}$. A VPS não pode ser utilizada como índice de enchimento ventricular esquerdo em pacientes portadores de edema pulmonar ou sob ventilação espontânea em conseqüência dos diferentes volumes correntes que ocorrem neste tipo de ventilação ${ }^{6}$. No choque hipovolêmico ocorre diminuição do débito cardíaco e aumento da resistência vascular sistêmica. Nessa situa- 
ção, as modificações do retorno venoso, provocadas pela ventilação artificial, serão maiores. Demonstrou-se em pacientes com sepse e sob tratamento com catecolaminas, que o componente ddown da VPS é um indicador importante da resposta do débito cardíaco à infusão de volume ${ }^{29}$. AVPS e o ddown apresentam alterações mais importantes durante a hipovolemia absoluta do que durante a hipotensão arterial provocada pelo nitroprussiato de sódio ${ }^{2}$. A VPS também não pode ser interpretada na presença de disritmias arrítmicas, sendo que o ritmo nodal pode aumentar a VPS por reduzir a pré-carga efetiva.

Após a reposição volêmica, alguns atributos hemodinâmicos retornaram aos valores iniciais de controle, como PAD, PAP, IRVP e ITSVE. Entretanto, alguns atributos permaneceram em valores inferiores aos do controle, como a PAM, PAPO e IRVS. Já outros valores hemodinâmicos permaneceram em valores acima do controle, como IC, IS e ITSVD. Deve-se ressaltar que o aumento importante do IC é uma das características da ação da solução hiperosmolar ${ }^{10}$.

A VPS e o ddown permaneceram em valores superiores aos do controle, indicando que a reposição volêmica com solução hiperosmótica e hiperoncótica, no volume utilizado (4 $\mathrm{ml} . \mathrm{kg}^{-1}$ ), melhorou mas não normalizou a volemia. O fato de vários cães apresentarem, após a reposição volêmica, VPS constituídas apenas de ddown, reforçam a idéia de que os animais ainda se encontravam em hipovolemia.

A expansão plasmática com SHD determina rápida diluição da hemoglobina e das proteínas plasmáticas do sangue ${ }^{30}$, como se verificou em relação à hemoglobina. Enquanto a solução hiperosmolar de cloreto de sódio a 7,5\% expande o volume plasmático pela mobilização de água celular, a adição de dextran 70, um colóide hiperoncótico, a essa solução aumenta o poder de retenção da água dentro do vaso com grande expansão do volume plasmático ${ }^{31}$. Embora a infusão de SHD em animais submetidos à hemorragia aumente rapidamente o volume plasmático, o volume sangüíneo permanece abaixo do normal ${ }^{17,31}$. Isto pode explicar, em nossa pesquisa, a manutenção da VPS e do ddown em valores inferiores aos do controle após a infusão de SHD. Deve-se ressaltar que a solução de dextran 70 empregada no estudo apresentou concentração $(3,75 \%)$ inferior à normalmente utilizada (6\%), o que pode ter também influenciado o resultado obtido.

Estudo sobre a pré-carga ventricular, por meio da VPS e da ecocardiografia transesofagiana, em 21 pacientes submetidos à cirurgia da aorta, e que se encontravam na Unidade de Terapia Intensiva sob sedação e ventilação, mostrou que as dimensões do ventrículo esquerdo no final da diástole correlacionaram-se bem com a magnitude da VPS $(r=0,80)$ e de ddown $(r=0,83)$. Após expansão volêmica, realizada em dois períodos com albumina humana a $5 \%$ (250 ml), houve correlação linear significante do ddown com a área diastólica final do ventrículo esquerdo (EDa) $(r=0,63)$ e com o índice cardíaco (IC) $(r=0,55)$. Assim, quanto maior o valor inicial do ddown, maior foi a alteração do EDa e do IC após a reposição volêmica. Segundo os autores, esses resultados demonstram que a análise da VPS fornece valiosas informações so- bre a pré-carga e a resposta à expansão de volume do ventrículo esquerdo ${ }^{5}$.

Na presente pesquisa, a infusão de solução hiperosmótica associada à solução hiperoncótica determinou, pelo efeito da primeira solução, aumento significante da osmolalidade plasmática, por causa do aumento do cloro e sódio plasmático. Geralmente a osmolalidade plasmática e a concentração de sódio e cloro retornam aos níveis de controle após 24 horas de administração da solução hiperosmolar. Embora não haja relato de efeitos adversos, pacientes sob uso de solução hipertônica necessitam monitoração dos níveis de sódio.

Em pacientes sob anestesia submetidos à hipovolemia, por retirada de $1000 \mathrm{ml}$ de sangue, observou-se que a reposição com o mesmo volume de solução de amido reduziu a VPS e ddown para valores inferiores aos do controle ${ }^{6}$. Vários autores observaram que a associação da solução hiperosmótica com solução hiperoncótica $(60 \mathrm{mmHg}$ ) resulta em maior aumento do volume plasmático, da pressão arterial e do débito cardíaco, todos dose-dependentes da solução de dextran 17,19,32-34 ou de amido ${ }^{6}$. Recentemente, demonstrou-se que parte da eficiência da solução hiperosmótica e hiperoncótica na expansão volêmica deve-se à mobilização de proteínas intestinais para a circulação, em proporção maior que à da solução fisiológica ${ }^{35}$.

O ddown, que durante a hipovolemia é praticamente responsável por toda a VPS ${ }^{1,6}$, após a administração de líquidos (cristalóides ou colóides) diminui ${ }^{1,5-7,29}$, enquanto durante a hipervolemia e/ou insuficiência cardíaca congestiva ele praticamente desaparece ${ }^{3}$. Assim, na presença de insuficiência circulatória, o ddown pode também diferenciar a disfunção miocárdica da inadequação da pré-carga. Uma curva de função do ventrículo esquerdo elevada (boa responsividade ao volume) se associa ao aumento do ddown enquanto a horizontalização dessa curva (má responsividade) se associa à diminuição do ddown ${ }^{36}$.

Resultados semelhantes foram obtidos em pacientes com sepse e alteração miocárdica que, em resposta à rápida expansão volêmica, apresentaram aumento relativo do dup ${ }^{29}$. Esse aspecto reforça a importância de medidas isoladas de ddown e dup, além da VPS. A interpretação da elevação da VPS como sinal de hipovolemia quando a mesma é caracterizada por dup dominante sobre ddown, pode levar à administração injustificada de líquidos.

A infusão de SHD não foi desprovida de efeitos colaterais, como o ocorrência de acidose hiperclorêmica em decorrência da hipernatremia e hipercloremia, além da solução infundida apresentar $\mathrm{pH}$ ao redor de 5,0. O aumento da $\mathrm{PaCO}_{2} \mathrm{e}$ $\mathrm{P}_{\mathrm{ET}} \mathrm{CO}_{2}$, decorrente do grande aumento do IC que se seguiu a infusão da solução, certamente também contribuiu para a acidose.

Concluindo, no cão, nas condições experimentais empregadas, a VPS e principalmente sua derivada ddown são indicadoras precoces de hipovolemia e guias sensíveis da reposição volêmica, sendo superiores a alguns índices hemodinâmicos, como a pressão da artéria pulmonar ocluída e o índice cardíaco. 


\section{Systolic Pressure Variation as an Earlier Hypovolemia Indicator and a Guide for Volume Replacement with Hypertonic and Hyperoncotic Solution in Dogs}

Odilar de Paiva Filho, TSA, M.D., José Reinaldo Cerqueira Braz, TSA, M.D., Fredson de Paula e Silva, M.D., Tiago Otávio Pedro, M.D., Paulo do Nascimento Júnior, TSA, M.D.

\section{INTRODUCTION}

Hypovolemia secondary to hemorrhage or fluid losses to the third space are difficult to be detected in the perioperative period because their most common clinical and hemodynamic signs have low specificity. Experimental studies have introduced a new method for preload evaluation based on the analysis of systolic blood pressure variation during mechanical ventilation ${ }^{1-4}$. The increased intra-thoracic pressure during mechanical ventilation inbreathing period decreases venous return and, as a consequence, blood pressure due to end right ventricle diastolic volume decrease. So, systolic pressure variation (SPV) corresponds to the difference between upper and lower systolic blood pressure obtained during one mechanical ventilation respiratory cycle with fixed tidal volume. A brief apnea allows systolic pressure variation to be nulled and to define its reference value, as from which it is possible to individualize SPV delta down (ddown) variants expressed in $\mathrm{mmHg}$ and corresponding to the difference between systolic blood pressure reference and lowervalue, and delta up (dup) which is the difference between systolic blood pressure reference and upper value (Figure 1).

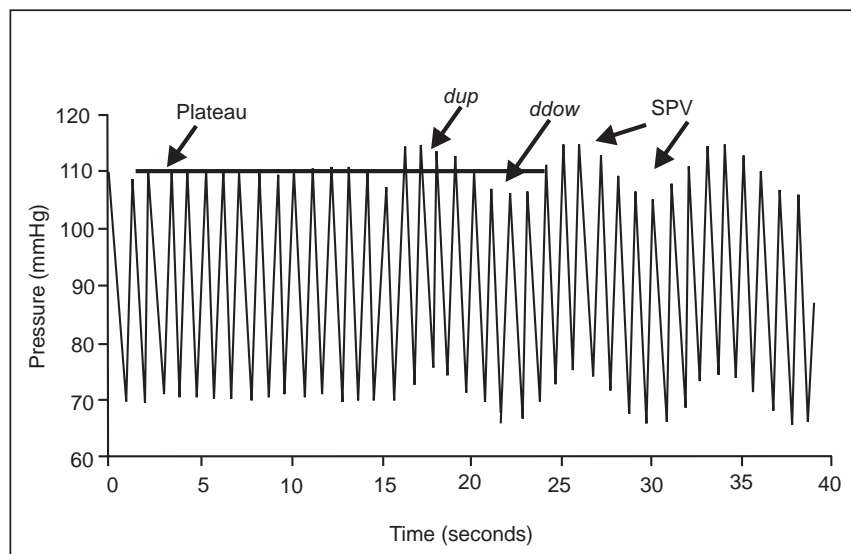

Figure 1 -Systolic pressure variation (SPV) is calculated as the difference between the highest and lowest systolic pressure value during the last respiratory cycle of first respiratory cycle after a phase of apnea (plateau). SPV is divided in two segments: delta up (dup) and delta down (ddown): dup is the difference between the highest systolic pressure and the plateau phase systolic pressure (apnea) and ddown is the difference between plateau phase systolic pressure and the lowest systolic pressure value

Revista Brasileira de Anestesiologia

Vol. 53, № 3, Maio - Junho, 2003
In men, SPV and ddown are good indicators for preload estimates ${ }^{4-8}$. The study of SPV and its components ddown and dup has shown that those attributes may help in volume replacement evaluation, according to left ventricle response $e^{5,6}$. During the $1980 \mathrm{~s}$, the concept of resuscitation using low $7.5 \%$ sodium chloride hypertonic solution volumes was introduced in the clinical practice. Whereas this clinical application was already known several decades ago, its clinical use only became popular as from 1980, when Velasco et al. ${ }^{9}$ have reported major survival of dogs submitted to hemorrhagic shock after receiving $7.5 \%$ sodium chloride in a volume corresponding to $10 \%$ of total removed blood.

The infusion of low hypertonic saline volumes ( 3 to $6 \mathrm{ml} . \mathrm{kg}^{-1}$ ) promptly increases cardiovascular and metabolic functions by combining plasma volume expansion with fast movement of interstitial and intracellular fluid to the vascular compartment, systemic vasodilatation and myocardial performance increase ${ }^{7,9,10}$.

In addition to the benefits in treating shock, hypertonic saline is effective to treat hypovolemia associated to major surgical procedures, such as aortic aneurysm correction ${ }^{11}$ and myocardial revascularization ${ }^{12-14}$.

The addition of dextran 70 to the hypertonic solution, described for the first time by Smith et al. (1985) ${ }^{15}$, increases volume expansion duration and intensity without losing the hemodynamic effect ${ }^{16}$. While the hypertonic solution expands the vascular space by mobilizing water from the cells, including red cells, the addition of a hyperoncotic colloid may selectively retain more water within the vessel. As a consequence, the association of both solutions increases cardiac output and survival rate after hemorrhagic shock, as compared to hypertonic solution alone ${ }^{17}$. Similar results were obtained with the addition of amide solutions to $7.5 \%$ sodium chloride ${ }^{18}$

Christ et al (1997) ${ }^{19}$ have suggested that hypertonic saline associated to $6 \%$ dextran 70 , which is hyperoncotic, opens new perspectives to handle perioperative fluids in elective and emergency abdominal aorta aneurysm surgeries. So, together with good hemodynamic stability, fluid balance is maintained less positive than during volume expansion with lactated Ringer's or amide solutions, thus decreasing the chances for edema. In addition, there seems to be positive microcirculatory effects of the associated solution, which may be highly beneficial in very high risk patients, such as decreased post-ischemia endothelial edema and post-capillary leucocyte adhesiveness, and increased tissue perfusion 20,21

In reviewing the literature, we have found no domestic or international research correlating SPV to the use of hypertonic saline associated or not to dextran in hypovolemic shock during anesthesia.

Our experimental research aimed at evaluating the importance of SPV, its derived ddown and dup, and of cardiovascular hemodynamic indices as early hypovolemia indicators and guides for volume replacement in dogs submitted to hypovolemia and volume replacement with $7.5 \%$ sodium chloride associated to $3.75 \%$ dextran 70 . 


\section{METHODS}

After local Animal Research Ethics Committee approval, 12 adult mongrel dogs, weighing 19 to $29 \mathrm{~kg}$ were used. After anesthetic induction with intravenous propofol $\left(6 \mathrm{mg} \cdot \mathrm{kg}^{-1}\right)$ and fentanyl $\left(5 \mu \mathrm{g} \cdot \mathrm{kg}^{-1}\right)$, the animals were positioned on Claude Bernard trough and submitted to tracheal intubation and mechanical ventilation with tidal volume of $20 \mathrm{ml}_{\mathrm{kg}}^{-1}$ and respiratory rate of 12 to $15 \mathrm{mov} \cdot \mathrm{min}^{-1}$, using a circuit with $\mathrm{CO}_{2}$ absorber and an Ohmeda Anesthesia machine model Excel 210 SE (USA) and biomonitor AS3 from Datex Ohmeda (Finland). Initially, anesthesia was maintained with $2.7 \%$ isoflurane (2 Minimum Alveolar Concentrations - MAC for the dog) using and Ohmeda (USA) gagged vaporizer and control of inspired and expired fractions with the gas, halogenate and ventilation analysis module. The following monitoring were installed: 3-channel ECG ( $D_{\|}$lead), thermometer sensor in lower esophagus, inspired and expired gases analyzer sensor and capnography close to the respiratory circuit $Y$ valve, and pulse oximetry with forceps-like sensor placed on animal's tongue.

Animals body surface was warmed with heated air between $42-46 \stackrel{\circ}{\mathrm{C}}$ inflated through a specific blanket using Mallinckrodt 's WarmTouch device (USA). Right femoral vein (RFV) was dissected and a polyethylene PE 240 catheter was inserted for the administration of initial $\left(0.6 \mathrm{mg}^{\mathrm{kg}}{ }^{-1}\right)$ and maintenance $\left(10 \mu \mathrm{g} . \mathrm{kg} \cdot \mathrm{min}^{-1}\right)$ rocuronium doses and volume replacement at the right time. Left femoral vein was dissected and a catheter was inserted for lactated Ringer's solution infusion ( $\left.5 \mathrm{ml} . \mathrm{kg}^{-1} \cdot \mathrm{min}^{-1}\right)$ through Abbott's Anne 2-channel continuous infusion pump (USA).

Right femoral artery was dissected and catheterized with PE 240 polyethylene catheter for hemorrhage and left femoral artery was dissected and catheterized to determine mean blood pressure and for blood collection for $\mathrm{pH}$ and blood gases analysis in the Chiron Diagnostics' device Rapidlab 865 (UK). Right external jugular vein was dissected and catheterized with an 8.5 F introducer and insertion of $7 \mathrm{~F}$ Swan-Ganz catheter in the pulmonary vein to measure cardiac output by thermodilution and pressures, in addition to blood collection for plasma osmolality, hemoglobin, sodium and chlorine analysis in the Chiron Diagnostics' device Rapidlab 865 (UK). Then, animals were submitted to splenectomy via median laparotomy.

After preparation, isoflurane expired MAC was decreased to $1.4 \%$ (1 MAC for the dog), for a10-minute period of hemodynamic stabilization. Then, mean blood pressure (MBP), pulmonary pressure (PP), right atrium pressure (RAP), pulmonary capillary wedge pressure (PCWP) and SPV values were recorded during ventilation and respiratory pause (10 seconds). Mixed arterial and venous blood were also collected and hemodynamic ventilation, oxygenation and temperature parameters were recorded (control moment). Next, four successive withdrawals of $5 \%\left(3.5 \mathrm{ml}^{\mathrm{kg}} \mathrm{kg}^{-1}\right)$ of total blood volume were performed ( $7 \%$ of body weight), in a total of $5 \%$ (moment $\mathrm{S}_{5}$ ), $10 \%$ (moment $\left.\mathrm{S}_{10}\right), 15 \%$ (moment $\mathrm{S}_{15}$ ) and $20 \%$ (moment $\mathrm{S}_{20}$ ) of animal's blood volume, and hemodynamic, ventilation, oxygenation and temperature parameters were recorded in addition to mixed arterial and venous blood collection for the analysis of the attributes immediately after each hemorrhage. Then, volume was replaced in the RFV with $7.5 \%$ sodium chloride associated to $3.75 \%$ dextran 70 (SHD) $\left(4 \mathrm{ml}^{\mathrm{kg}}{ }^{-1}\right)$ in a total time of 10 minutes using Arcomed's peristaltic infusion pump (Switzerland). Five (moment $R V_{5}$ ) and 30 (moment $R V_{30}$ ) minutes after volume replacement completion attributes were recorded and samples of mixed arterial and venous blood collected, followed by animals' euthanasia with excessive anesthesia (sodium pentobarbital).

Attributes studied were: Respiratory - tidal volume (TV $\left.\mathrm{ml} . \mathrm{kg}^{-1}\right)$, respiratory rate $\left(\mathrm{RR}-\mathrm{mov} \cdot \mathrm{min}^{-1}\right)$, inspiratory pressure (IP - $\left.\mathrm{cmH}_{2} \mathrm{O}\right)$, chest compliance $\left(\mathrm{CC}-\mathrm{ml}^{\mathrm{cm}} \mathrm{cm}^{-1} \mathrm{H}_{2} \mathrm{O}\right)$, end tidal $\mathrm{CO}_{2}\left(\mathrm{P}_{\mathrm{ET}} \mathrm{CO}_{2}-\mathrm{mmHg}\right)$ and hemoglobin peripheral saturation $\left(\mathrm{SpO}_{2}\right.$ - \%); Hemodynamic - heart rate (HR beat. $\left.\mathrm{min}^{-1}\right)$, mean blood pressure (MBP - $\mathrm{mmHg}$ ), mean pulmonary artery pressure (MPAP - $\mathrm{mmHg}$ ), mean right atrium pressure (MRAP - $\mathrm{mmHg}$ ), pulmonary capillary wedge pressure $(\mathrm{PCWP}-\mathrm{mmHg})$ cardiac index $\left(\mathrm{Cl}-\mathrm{L} \cdot \mathrm{min}^{-1} \cdot \mathrm{m}^{-2}\right)$, systolic index (SI- ml.beat $\left.{ }^{-1} \cdot \mathrm{m}^{-2}\right)$, systemic vascular resistance index (SVRI - dyne.s. $\mathrm{cm}^{-5} \cdot \mathrm{m}^{-2}$ ), pulmonary vascular resistance index (PVRI - dyne.s. $\mathrm{cm}^{-5} \cdot \mathrm{m}^{-2}$ ), left ventricle stroke work (LVSW - g.min. $\mathrm{m}^{-2}$ ), right ventricle stroke work (RVSW g. min. $\mathrm{m}^{-2}$ ), systolic pressure variation (SPV - $\mathrm{mmHg}$ ), delta up (dup - mmHg), delta down (ddown - mmHg); Blood - hemoglobin $\left(\mathrm{Hb}-\mathrm{mg} \cdot \mathrm{dL}^{-5}\right)$, plasma sodium $\left(\mathrm{Na}^{+}-\mathrm{mMol} \cdot \mathrm{L}^{-3}\right)$, plasma chlorine $\left(\mathrm{Cl}^{-}\right.$- $\left.\mathrm{mMol} . \mathrm{L}^{-1}\right)$, plasma osmolality (Oplasm mOsm. $\left.\mathrm{kg}^{-1} \mathrm{H}_{2} \mathrm{O}\right)$, arterial $\mathrm{pH}(\mathrm{pHa})$ and arterial partial carbon dioxide pressure $\left(\mathrm{PaCO}_{2}-\mathrm{mmHg}\right)$; Temperature - esophageal $\left(\mathrm{T}_{\text {Esoph }}-{ }^{\circ} \mathrm{C}\right)$. SHD was prepared with the association of $20 \%$ sodium chloride $(37.5 \mathrm{ml})$ and $6 \%$ dextran 70 in $5 \%$ glucose $(62.5 \mathrm{ml})$. This way, final solution $(100 \mathrm{ml})$ contained $7.5 \% \mathrm{NaCl}$ in $3.75 \%$ dextran 70 .

There is no monitoring system able to automatically calculate SPV. It is necessary to record blood pressure curve during some respiratory cycles and during an episode of apnea, using slow velocity in the printing paper (Figure 1). To determine indirectly obtained hemodynamic attributes a biomonitor AS3 software was used, with the entry of necessary attribute values.

\section{Statistical Analysis}

Since attributes distribution was non-parametric, Friedman's test was initially applied. Contrasts among group medians were evaluated by Student-Newman-Keuls test and data are presented as tables or figures with indications of attributes median, first (25\%) and third $(75 \%)$ quartiles. Pearson linear regression was used to determine the correlation of SPV, ddown and dup with other hemodynamic parameters. Significance level was considered $p<0.05$. 


\section{RESULTS}

Demographics data are shown in table I.

Table I - Demographics Data

\begin{tabular}{lccc}
\hline Weight $(\mathrm{kg})^{*}$ & Length $(\mathrm{cm})^{*}$ & Body surface $^{*}$ & Gender $(\mathrm{M} / \mathrm{F})$ \\
\hline $23.7 \pm 3,0$ & $107.4 \pm 6.6$ & $0.81 \pm 0.07$ & $11 / 1$ \\
\hline
\end{tabular}

* Values expressed in Mean \pm SD
Ventilatory attributes are shown in table II. There have been no significant differences in respiratory parameters, with the exception of $\mathrm{P}_{\mathrm{ET}} \mathrm{CO}_{2}$, which was significantly increased at final hemorrhage and even more after volume replacement, as compared to control.

Hemodynamic cardiovascular results are shown in tables III and IV and figures 2 and 3 . HR values, although high, were not significantly changed during hemorrhage and after volume replacement. Mean blood pressure has significantly de-

Table II - Values of Median, $1^{\text {st }}$ and $3^{\text {rd }}$ Quartiles of Tidal Volume (TV), Respiratory Rate (RR), Inspiratory Pressure (IP), Chest Compliance (CC), Carbon Dioxide End Expiratory Pressure $\left(\mathrm{P}_{\mathrm{ET}} \mathrm{CO}_{2}\right)$ and Peripheral Oxygen Hemoglobin Saturation $\left(\mathrm{SpO}_{2}\right)$

\begin{tabular}{|c|c|c|c|c|c|c|}
\hline \multirow[t]{2}{*}{ Moments } & \multicolumn{6}{|c|}{ Attributes } \\
\hline & TV $\mathrm{ml} . \mathrm{kg}^{-1}$ ) & $\mathrm{HR}\left(\mathrm{mov} \cdot \mathrm{min}^{-1}\right)$ & $\mathrm{PI}\left(\mathrm{cmH}_{2} \mathrm{O}\right)$ & $\mathrm{CC}\left(\mathrm{ml}^{\mathrm{c}} \mathrm{cm}^{-1} \mathrm{H}_{2} \mathrm{O}\right)$ & $\mathrm{P}_{\mathrm{ET}} \mathrm{CO}_{2}(\mathrm{mmHg})$ & $\mathrm{SpO}_{2}(\%)$ \\
\hline Control & $19.5[18.8 ; 20.4] \mathrm{a}^{(1)}$ & $15.5[10.5 ; 18.0] a$ & $15.0[14.0 ; 17.0] \mathrm{a}$ & $34.0[32.0 ; 41.5] \mathrm{a}$ & $34.0[33.5 ; 35.5] \mathrm{a}$ & $99.0[97.0 ; 100.0] \mathrm{a}$ \\
\hline $\mathrm{S}_{5}$ & $19.5[18.6 ; 20.2] a$ & $15.5[11.5 ; 18.0] a$ & $15.0[14.5 ; 17.0] a$ & $34.0[31.5 ; 42.0] a$ & $35.0[33.5 ; 36.0] a$ & $99.0[98.0 ; 100.0] \mathrm{a}$ \\
\hline$S_{10}$ & $19.5[18.6 ; 20.2] a$ & $15.5[11.5 ; 18.0] a$ & $15.0[14.5 ; 16.0] a$ & $33.5[31.0 ; 40.5] \mathrm{a}$ & $36.0[34.0 ; 36.0] a$ & $99.0[98.5 ; 100.0] a$ \\
\hline $\mathrm{S}_{15}$ & $19.5[18.6 ; 20.2] a$ & $15.5[11.5 ; 18.0] a$ & $15.0[14.5 ; 17.0] \mathrm{a}$ & $34.0[30.5 ; 41.5] \mathrm{a}$ & $36.0[33.5 ; 38.0] \mathrm{b}$ & $99.0[98.5 ; 100.0] a$ \\
\hline $\mathrm{RV}_{5}$ & $19.2[18.4 ; 19.6] a$ & $15.5[11.5 ; 18.0] a$ & $15.5[14.0 ; 17.0] a$ & $37.0[31.0 ; 41.5] a$ & $42.0[38.5 ; 44.5] \mathrm{d}$ & $99.0[97.5 ; 99.0] a$ \\
\hline $\mathrm{RV}_{30}$ & $19.1[18.6 ; 20.7] \mathrm{a}$ & $15.5[11.5 ; 18.0] a$ & $16.0[14.0 ; 16.5] a$ & $36.5[30.5 ; 42.0] a$ & $40.0[38.0 ; 43.5] \mathrm{d}$ & $99.0[98.0 ; 99.0] a$ \\
\hline
\end{tabular}

(1) Moments represented by medians followed by different small letters within the group are significantly different $(p<0.05)$

Table III - Values of Median, $1^{\text {st }}$ and $3^{\text {rd }}$ Quartiles of Heart Rate (HR), Mean Blood Pressure (MBP) Right Atrium Pressure (RAP), Pulmonary Artery Pressure (PAP), Pulmonary Capillary Wedge Pressure (PCWP) and Cardiac Index (Cl)

\begin{tabular}{|c|c|c|c|c|c|c|}
\hline \multirow[t]{2}{*}{ Moments } & \multicolumn{6}{|c|}{ Attributes } \\
\hline & HR (beat. $\mathrm{min}^{-1}$ ) & $\mathrm{MBP}(\mathrm{mmHg})$ & $\mathrm{RAP}(\mathrm{mmHg})$ & $\mathrm{PAP}(\mathrm{mmHg})$ & PCWP (mmHg) & $\mathrm{Cl}\left(\mathrm{L} \cdot \mathrm{min}^{-1} \cdot \mathrm{m}^{-2}\right)$ \\
\hline Control & $128.0[113 ; 145] a^{(1)}$ & $90.5[85.5 ; 99.0] \mathrm{c}$ & $3.0[3.0 ; 3.5] \mathrm{c}$ & $12.0[11.5 ; 15.0] \mathrm{d}$ & $5.0[4.0 ; 7.0] \mathrm{b}$ & $3.50[2.75 ; 4.30] \mathrm{a}$ \\
\hline $\mathrm{S}_{5}$ & $134.0[111 ; 147] \mathrm{a}$ & $80.5[77.0 ; 96.0] \mathrm{b}$ & $2.0[2.0 ; 3.0] \mathrm{b}$ & $12.0[10.5 ; 14.0] \mathrm{d}$ & $3.5[2.5 ; 6.0] \mathrm{a}$ & $3.70[2.75 ; 3.80] \mathrm{a}$ \\
\hline $\mathrm{S}_{10}$ & $135.0[119 ; 156] \mathrm{a}$ & $79.0[72.0 ; 84.0] \mathrm{b}$ & $2.0[1.5 ; 3.0] \mathrm{a}$ & $11.5[10.0 ; 14.0] \mathrm{c}$ & $3.5[2.5 ; 4.5] \mathrm{a}$ & $3.35[2.85 ; 3.80] \mathrm{a}$ \\
\hline $\mathrm{S}_{15}$ & 141.0[123;159]a & $73.0[71.0 ; 79.0] \mathrm{b}$ & $2.0[1.0 ; 2.5] a$ & $10.0[9.0 ; 12.5] \mathrm{b}$ & $2.0[2.0 ; 3.0] \mathrm{a}$ & $3.20[2.75 ; 3.65] \mathrm{a}$ \\
\hline $\mathrm{RV}_{5}$ & $143.0[128 ; 162] a$ & $79.0[75.5 ; 84.5] \mathrm{b}$ & $3.0[2.0 ; 3.0] \mathrm{d}$ & $13.011 .5 ; 14.0] \mathrm{d}$ & $3.0[2.0 ; 4.0] a$ & $5.05[4.40 ; 5.75] \mathrm{c}$ \\
\hline $\mathrm{RV}_{30}$ & $142.0[132 ; 163] \mathrm{a}$ & $83.5[71.5 ; 93.0] \mathrm{b}$ & $2.0[1.5 ; 2.0] \mathrm{c}$ & $12.5[11.5 ; 14.5] d$ & $3.0[2.0 ; 4.5] \mathrm{a}$ & $4.70[4.20 ; 5.30] \mathrm{b}$ \\
\hline
\end{tabular}

(1) Moments represented by medians followed by different small letters within the group are significantly different $(p<0.05)$

Table IV - Values of Median, $1^{\text {st }}$ and $3^{\text {rd }}$ Quartiles of Systolic Index (SI), Systemic Vascular Resistance Index (SVRI), Pulmonary Vascular Resistance Index (PVRI), Left Ventricle Stroke Work Index (LVSWI), Right Ventricle Stroke Work Index (RVSWI) and Delta up (dup)

\begin{tabular}{|c|c|c|c|c|c|c|}
\hline \multirow[t]{2}{*}{ Moments } & \multicolumn{6}{|c|}{ Attributes } \\
\hline & $\mathrm{SI}\left(\mathrm{ml}\right.$.beat $\left.{ }^{-1} \cdot \mathrm{m}^{-2}\right)$ & SVRI (dyne.s.cm ${ }^{-5} \cdot \mathrm{m}^{-2}$ ) & PVRI (dyne.s.cm ${ }^{-5} \cdot \mathrm{m}^{-2}$ ) & LVSWI (g.min.m ${ }^{-2}$ ) & RVSWI (g.min.m ${ }^{-2}$ ) & Dup (mmHg) \\
\hline Control & $28.0[24.5 ; 32.0] \mathrm{d}^{(1)}$ & 2133[1658;2679]b & $156[112 ; 244] \mathrm{b}$ & $35.3[29.1 ; 39.8] d$ & $3.20[2.40 ; 4.70] c$ & $2.0[0.0 ; 2.5] \mathrm{a}$ \\
\hline $\mathrm{S}_{5}$ & $27.0[22.5 ; 29.5] c$ & $1944[1656 ; 2489] \mathrm{b}$ & $147[131 ; 271] \mathrm{b}$ & $29.0[26.2 ; 35.6] \mathrm{c}$ & $3.20[2.60 ; 3.95] \mathrm{c}$ & $0.6[0.0 ; 1.5] a$ \\
\hline$S_{10}$ & $25.0[22.0 ; 28.0] \mathrm{b}$ & $1769[1675 ; 2184] \mathrm{b}$ & $169[137 ; 207] \mathrm{b}$ & $26.0[22.9 ; 29.8] \mathrm{b}$ & $3.05[2.05 ; 3.80] \mathrm{b}$ & $0.0[0.0 ; 1.4] \mathrm{a}$ \\
\hline $\mathrm{S}_{15}$ & $24.5[19.5 ; 29.5] a$ & $1776[1552 ; 2057] \mathrm{b}$ & $190[144 ; 263] \mathrm{b}$ & $27.1[19.6 ; 30.8] \mathrm{b}$ & $3.10[1.95 ; 3.60] \mathrm{b}$ & $0.0[0.0 ; 1.9] \mathrm{a}$ \\
\hline $\mathrm{RV}_{5}$ & $37.0[28.0 ; 40.5] f$ & $1259[1179 ; 1633] a$ & $140[119 ; 195] \mathrm{a}$ & $35.0[30.5 ; 44.5] \mathrm{e}$ & $4.80[3.80 ; 5.70] \mathrm{d}$ & $0.0[0.0 ; 0.1] \mathrm{a}$ \\
\hline $\mathrm{RV}_{30}$ & $31.5[26.5 ; 38.0] \mathrm{e}$ & $1369[1240 ; 1783] a$ & $149[132 ; 223] \mathrm{b}$ & $32.7[29.6 ; 42.4] \mathrm{d}$ & $4.40[3.55 ; 5.65] d$ & $0.6[0.0 ; 1.9] a$ \\
\hline
\end{tabular}

(1) Moments represented by medians followed by different small letters within the group are significant different $(p<0.05)$ 


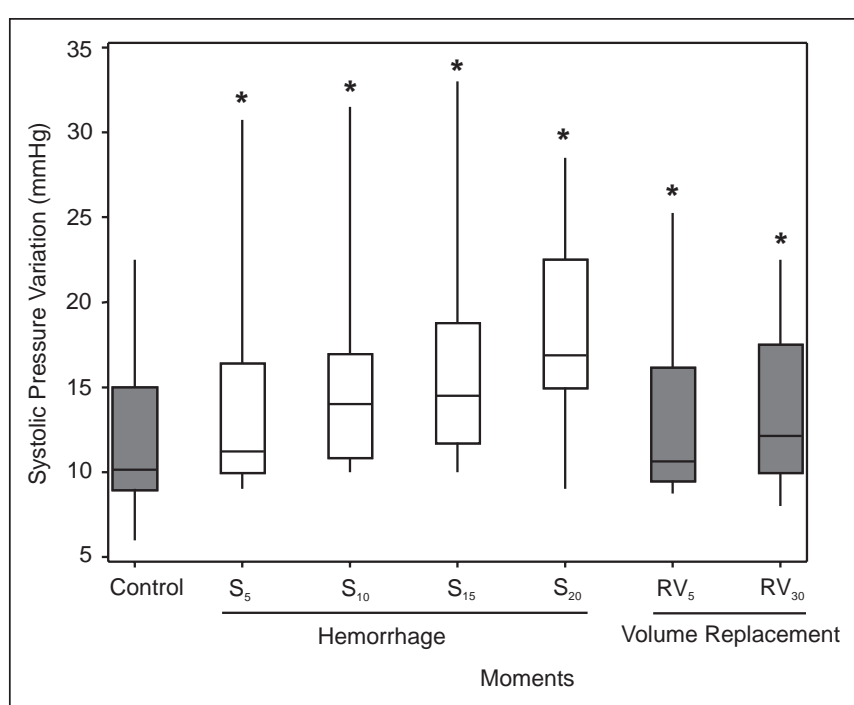

Figure 2 - Box Plot Presenting Minimum Values of $1^{\text {st }}$ Quartile, Median, $3^{\text {rd }}$ Quartile and Maximum Systolic Pressure Variation in Studied Moments

${ }^{*} \mathrm{p}<0.05: \mathrm{C}<\left(\mathrm{RV}_{5}=\mathrm{RV}_{30}=\mathrm{S}_{5}\right)<\mathrm{S}_{10}<\left(\mathrm{S}_{15}=\mathrm{S}_{20}\right)$

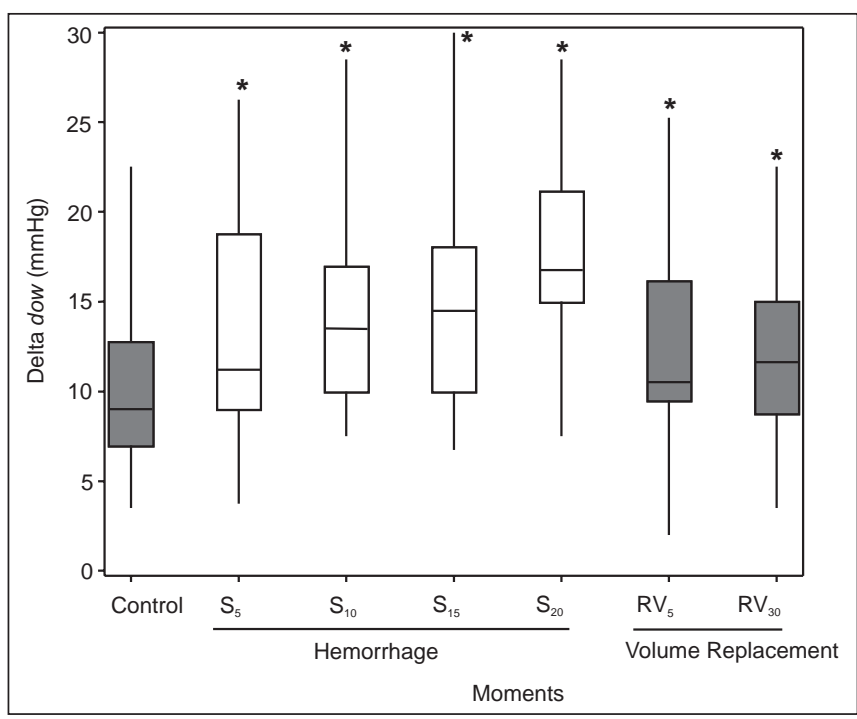

Figure 3 - Box Plot Presenting Minimum Values of $1^{\text {st }}$ Quartile, Median, $3^{\text {rd }}$ Quartile and Maximum ddown in Studied Moments ${ }^{*} \mathrm{p}<0.05: \mathrm{C}<\left(\mathrm{S}_{5}=\mathrm{RV}_{5}=\mathrm{RV}_{30}\right)<\mathrm{S}_{10}<\mathrm{S}_{15}<\mathrm{S}_{20}$

creased during hemorrhage and have significantly increased after volume replacement, however without reaching baseline values. Mean pulmonary artery pressure has significantly and progressively decreased during hemorrhage and have significantly increased after volume replacement, to values similar to baseline. Mean right atrium pressure has significantly decreased during hemorrhage and has significantly increased after volume replacement, to values initially higher than baseline and, after 30 minutes, to values similar to baseline. Pulmonary capillary wedge pressure has significantly decreased after the first hemorrhage and remained lower than baseline during remaining hemorrhage periods and volume replacement. Cardiac index has not significantly changed during hemorrhage, bur significantly increased after volume replacement to values above baseline.

SI has significantly and progressively decreased during hemorrhage and has significantly increased after volume replacement, to levels above baseline. IRVS and IRVP have not significantly changed during hemorrhage, but have significantly decreased after volume replacement and, in the final moment of the study $\left(\mathrm{RV}_{30}\right)$, IRVS was maintained in levels below baseline and IRVP was maintained in similar levels to baseline. ITSVE and ITSVD have significantly decreased during hemorrhage, but have significantly increased after volume replacement. In $R_{30}$, ITSVD was maintained above baseline and ITSVE was maintained in levels similar to baseline.

SPV and ddown have significantly and progressively increased during hemorrhage and have significantly decreased after volume replacement however maintaining levels above baseline (Figures 2 and 3). Dup has not significantly changed.

Plasma sodium and chloride levels have significantly increased after volume replacement (Figure 4 and Table V) causing a significant increase in plasma osmolality (Figure 5). Hb levels, which had progressively decreased after each hemorrhage, decreased even more after volume replacement (Table V). pHa has significantly decreased with hemorrhage and after volume replacement, while $\mathrm{PaCO}_{2}$ has significantly increased after volume replacement (Table V). $\mathrm{T}_{\text {esoph }}$ has not significantly changed (Table V).

Pearson's correlation of systolic pressure variation (SPV) and ddown with remaining hemodynamic attributes studied along the experiment, with indication of values $r$ and $p$, are shown in table VI.

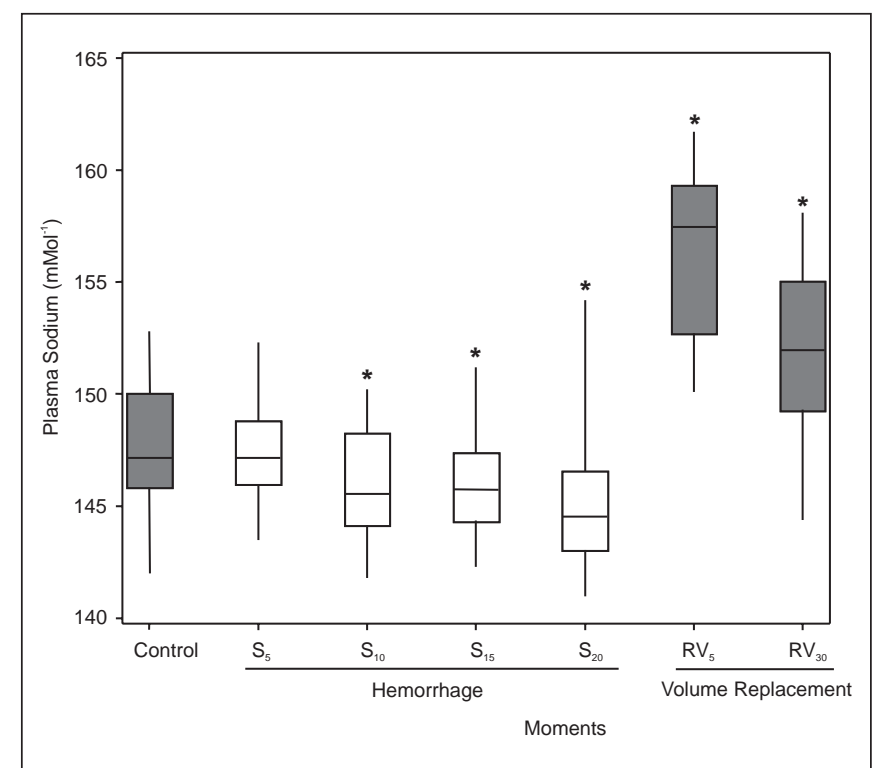

Figure 4 - Box Plot Showing Minimum, $1^{\text {st }}$ Quartile, Median, $3^{\text {rd }}$ Quartile and Maximum Plasma Sodium Volumes in Studied Moments

${ }^{*} \mathrm{p}<0.05:\left(\mathrm{S}_{10}=\mathrm{S}_{15}=\mathrm{S}_{30}\right)<\left(\mathrm{C}=\mathrm{S}_{5}\right)<\mathrm{RV}_{30}<\mathrm{RV}_{5}$

Revista Brasileira de Anestesiologia Vol. 53, № 3, Maio - Junho, 2003 
Table V - Values of Median, $1^{\text {st }}$ and $3^{\text {rd }}$ Quartiles of Hemoglobin $(\mathrm{Hb})$, Plasma Chlorine $\left(\mathrm{Cl}^{-}\right)$, arterial pH (pHa), Carbon dioxide End Expiratory Pressure $\left(\mathrm{PaCO}_{2}\right)$ and Esophageal Temperature $\left(\mathrm{T}_{\text {Esoph }}\right)$

\begin{tabular}{|c|c|c|c|c|c|}
\hline \multirow[t]{2}{*}{ Moments } & \multicolumn{5}{|c|}{ Attributes } \\
\hline & $\mathrm{Hb}\left(\mathrm{mg} \cdot \mathrm{dl}^{-1}\right)$ & $\mathrm{Cl}-\left(\mathrm{mOsm} \cdot \mathrm{L}^{-1}\right)$ & $\mathrm{pHa}$ & $\mathrm{PaCO}_{2}(\mathrm{mmHg})$ & Tesoph $\left({ }^{\circ} \mathrm{C}\right)$ \\
\hline Control & $13.0[12.3 ; 14.2] \mathrm{g}^{(1)}$ & $110.5[109.0 ; 112.0] a$ & $7.38[7.33 ; 7.45] \mathrm{d}$ & $36.2[34.8 ; 38.6] \mathrm{a}$ & $38.3[37.7 ; 39.3] a$ \\
\hline $\mathrm{S}_{5}$ & 13.3[12.5;13.9]f & $110.0[108.5 ; 112.0] \mathrm{a}$ & $7.38[7.31 ; 7.44] \mathrm{c}$ & $38.6[35.7 ; 39.9] a$ & $38.3[37.7 ; 39.1] \mathrm{a}$ \\
\hline$S_{10}$ & $12.3[11.9 ; 13.6] \mathrm{e}$ & $110.0[107.5 ; 112.0] a$ & $7.38[7.32 ; 7.41] \mathrm{c}$ & $37.5[36.5 ; 42.5] \mathrm{a}$ & $38.2[37.7 ; 39.1] \mathrm{a}$ \\
\hline $\mathrm{S}_{15}$ & $12.5[11.9 ; 13.5] \mathrm{d}$ & $110.5[108.0 ; 112.0] \mathrm{a}$ & $7.36[7.30 ; 7.42] \mathrm{c}$ & $40.1[36.5 ; 43.5] a$ & $38.2[37.7 ; 39.0] a$ \\
\hline $\mathrm{RV}_{5}$ & $10.9[10.1 ; 11.1] \mathrm{a}$ & $122.5[121.0 ; 125.5] \mathrm{c}$ & $7.30[7.23 ; 7.34] a$ & $46.0[41.1 ; 49.6] \mathrm{b}$ & $38.2[37.8 ; 38.8] \mathrm{a}$ \\
\hline $\mathrm{RV}_{30}$ & $11.1[10.6 ; 11.3] a$ & $119.5[117.0 ; 122.5] \mathrm{b}$ & 7.30[7.25;7.38]a & $44.5[39.8 ; 48.1] \mathrm{b}$ & $38.3[37.8 ; 38.7] \mathrm{a}$ \\
\hline
\end{tabular}

${ }^{(1)}$ Moments represented by medians followed by different small letters within the group are significantly different $(p<0.05)$

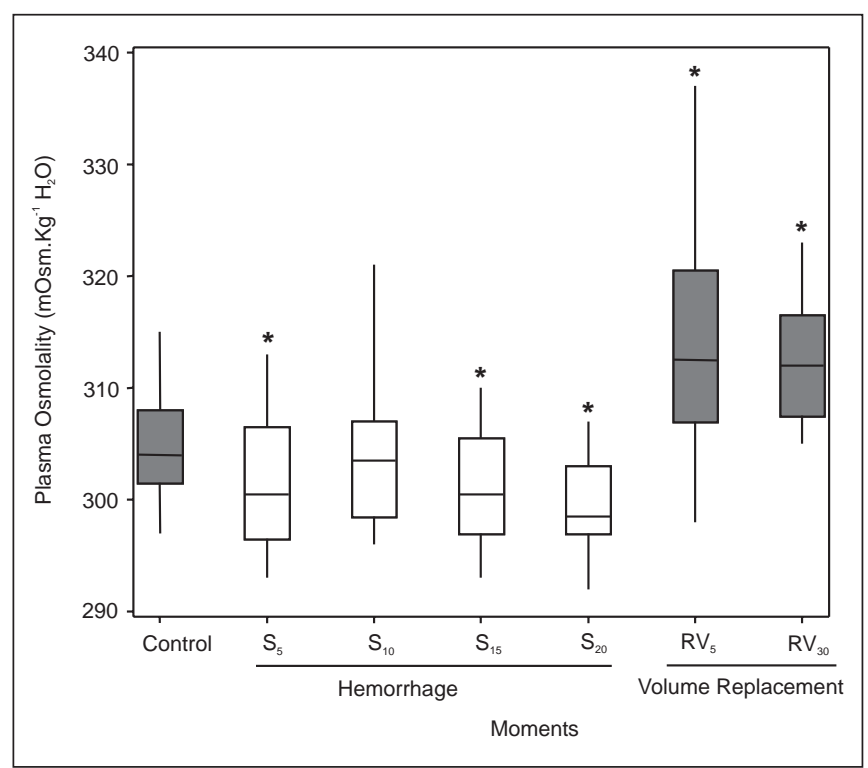

Figure 5 - Box Plot showing Minimum, $1^{\text {st }}$ quartile, Median, $3^{\text {rd }}$ Quartile and Plasma Osmolality in Studied Moments * $\mathrm{p}<0,05: \mathrm{S}_{20}<\left(\mathrm{S}_{5}=\mathrm{S}_{15}\right)<\left(\mathrm{C}=\mathrm{S}_{10}\right)<\left(\mathrm{RV}_{5}=\mathrm{RV}_{30}\right)$

Correlation between SPV and ddown was high. The correlation of those attributes with remaining hemodynamic attributes was mostly significant. Higher SPV correlations were, in decreasing order, with: ddown, SI, ITSVE, PCWP, PAP, CI and ITSVD, while higher ddown correlations were, in decreasing order, with SPV, PCWP, SI, DBP and ITSVE. Dup had its higher correlations with PCWP and DBP.

Analyzing Pearson's correlation in each moment, it was observed a significant and high correlation in all moments ( $r$ always above 0.90 ) between SPV and ddown. The only significant correlation in the control group was between ddown and PCWP. During hemorrhages, SPV and ddown had significant correlations with SI, ITSVE and PCWP, in addition to $\mathrm{Cl}$ in $\mathrm{RV}_{30}$. After volume replacement, there has been a significant correlation of SPV and ddown with SI and PCWP, while ddown alone had a significant interaction also with DBP and ITSVE.

Revista Brasileira de Anestesiologia

Vol. 53, № 3, Maio - Junho, 2003
Table VI - Pearson's Correlation Coefficient of Systolic Pressure Variation (SPV), ddown and dup with Remaining Hemodynamic Attributes throughout the Experiment $(n=84)$, with Indication of $r$ and $p$ values

\begin{tabular}{|c|c|c|c|}
\hline Attributes & SPV & ddown & dup \\
\hline Mean blood pressure & $\begin{array}{l}r=-0.23751 \\
p=0.0296\end{array}$ & $\begin{array}{l}r=-0.20551 \\
p=0.0613\end{array}$ & $\begin{array}{l}r=-0.07062 \\
p=0.5233\end{array}$ \\
\hline Cardiac index & $\begin{array}{l}r=-0.43758 \\
p=0.0001\end{array}$ & $\begin{array}{l}r=-0.36921 \\
p=0.0005\end{array}$ & $\begin{array}{l}r=-0.21172 \\
p=0.0532\end{array}$ \\
\hline $\begin{array}{l}\text { Pulmonary artery } \\
\text { presssure }\end{array}$ & $\begin{array}{l}r=-0.22916 \\
p=0.0360\end{array}$ & $\begin{array}{l}r=-0.25773 \\
p=0.0179\end{array}$ & $\begin{array}{l}r=0.15129 \\
p=0.1695\end{array}$ \\
\hline Right atrium pressure & $\begin{array}{l}r=-0.44495 \\
p=0.0001\end{array}$ & $\begin{array}{l}r=-0.53651 \\
p=0.0001\end{array}$ & $\begin{array}{l}r=0.41326 \\
p=0.0001\end{array}$ \\
\hline $\begin{array}{l}\text { Pulmonary capillary } \\
\text { wedge pressure }\end{array}$ & $\begin{array}{l}r=-0.47252 \\
p=0.0001\end{array}$ & $\begin{array}{l}r=-0.57140 \\
p=0.0001\end{array}$ & $\begin{array}{l}r=0.43389 \\
p=0.0001\end{array}$ \\
\hline Systolic index & $\begin{array}{l}r=-0.59646 \\
p=0.0001\end{array}$ & $\begin{array}{l}r=-0.56753 \\
p=0.0001\end{array}$ & $\begin{array}{l}r=-0.03708 \\
p=0.7377\end{array}$ \\
\hline RV stroke work index & $\begin{array}{l}r=-0.40938 \\
p=0.0001\end{array}$ & $\begin{array}{l}r=-0.36932 \\
p=0.0001\end{array}$ & $\begin{array}{l}r=-0.09989 \\
p=0.3660\end{array}$ \\
\hline LV stroke work index & $\begin{array}{l}r=-0.54229 \\
p=0.0001\end{array}$ & $\begin{array}{l}r=-0.49679 \\
p=0.0001\end{array}$ & $\begin{array}{l}r=-0.09982 \\
p=0.3663\end{array}$ \\
\hline $\begin{array}{l}\text { Systemic vascular } \\
\text { resistance index }\end{array}$ & $\begin{array}{l}r=0.33201 \\
p=0.0020\end{array}$ & $\begin{array}{l}r=0.27765 \\
p=0.0001\end{array}$ & $\begin{array}{l}r=0.18839 \\
p=0.0861\end{array}$ \\
\hline SPV & - & $\begin{array}{l}r=0.96708 \\
p=0.0001\end{array}$ & $\begin{array}{l}r=-0.00327 \\
p=0.9765\end{array}$ \\
\hline Ddown & $\begin{array}{l}r=0.96708 \\
p=0.0001\end{array}$ & - & $\begin{array}{l}r=-0.24955 \\
p=0.0221\end{array}$ \\
\hline
\end{tabular}

\section{DISCUSSION}

Medium to large size dogs were used for having hemodynamic parameters compatible to humans and an adequate size to stand surgical preparation used in the research to help hemodynamic monitoring through a catheter inserted in the pulmonary artery.

Tidal volume, inspiratory pressure and compliance maintained in uniform levels along the experiment was important, because SPV, ddown and dup are influenced by such attributes. Tidal volume used was similar to that of the awaken dog $\left(20 \mathrm{ml}^{\mathrm{kg}} \mathrm{kg}^{-1}\right)^{22}$. Perel et al. $(1987)^{1}$ have used a tidal volume of $12 \mathrm{ml} . \mathrm{kg}^{-1}$ and inflatable vest around dogs chest to maintain chest compliance similar to that of humans. In this and in our study, where no inflatable vest was used in the dog, chest 
compliance was similar to humans. Different tidal volumes used in the two studies may explain results obtained.

Circulating blood flow in the adult and healthy dog is $7 \%$ of its body weight ${ }^{1}$ and blood volume removed from each animal was $14 \mathrm{ml} . \mathrm{kg}^{-1}$ determining hypovolemia degrees I to II (Committee on Trauma ${ }^{23}$. Blood removal in four periods at 15-minute intervals has surely prevented major cardiocirculatory changes because it allowed the animal to compensate, at least partially, volume loss.

Both plasma and red cells volume are partially restored during early hemorrhagic shock (degree I), but the intensity of such adjustment varies among individuals and species.

The activation of the sympathetic system leads to capacitance vessels constriction, causing major central mobilization of the blood located in large and small veins, thus increasing preload. In this stage, there is also arterial vasoconstriction with restricted flow to skin, muscle-skeletal, renal and splancnic territories, while there is a locally-mediated vasodilatation in brain and myocardium.

Plasma volume recovery is a result of pre-capillary selective vasoconstriction that decreases microcirculation hydrostatic pressure. As a result, oncotic pressure predominates with interstitial fluid absorption. This phenomenon is known as internal self-transfusion or transcapillary shift, which compensates plasma losses and tends to cause hemodilution ${ }^{24}$.

Circulating red cells volume replacement is also dependent on sympathetic activation with splenoconstriction, which is very efficient in dogs due to their large spleen, and is able to transfer to circulation blood reserves, with high hematocryt accumulated in the splancninc parenchyma.

Splenectomy, to better control volume during hemorrhage, has caused relative hypovolemia in the animals, considering that volume replacement with lactated Ringer's in this period was low $\left(5 \mathrm{ml} \cdot \mathrm{kg}^{-1} \cdot \mathrm{h}^{-1}\right)$. The fact that SPV in many dogs consisted only of ddown at control moment, reinforces this idea. Other hemodynamic attributes studied were within normal ranges for anesthetized dogs ${ }^{22}$, thus further reinforcing the importance of SPV and ddown as early indicators of hypovolemia. During hemorrhage and hypovolemia, as observed in our research, ddown may significantly increase becoming responsible for almost all SPV ${ }^{1,6}$. Normal ddown values are 5 to $6 \mathrm{mmHg}$, normal dup values are 2 to $4 \mathrm{mmHg}$ and normal SPV values are 8 to $10 \mathrm{mmHg}^{5,6}$.

Insidious perioperative hypovolemia is difficult to detect and some clinically used attributes, such as HR and MBP, are not always good indicators of volume changes, as it was observed in this research. Even PCWP, considered significant ${ }^{12}$, has limitations as indicator of left ventricle end diastolic volume ${ }^{5,25,26}$.

In our research, during hemorrhage, ddown was the attribute with the best correlation with each hemorrhage level, together with hemoglobin. Other attributes with good correlation in each hemorrhage stage were: SPV, PAP, SI and ITSVD. Some attributes had no correlation with hemorrhage levels, such as HR, CI, IRVS, IRVP and dup.
So, both SPV and ddown are sensitive hypovolemia indicators in dogs and humans ${ }^{5-7,27}$.

During ventilation inspiratory phase, the increase in right atrium intrathoracic and intraluminal pressure increases venous return resistance decreasing right atrium preload and right ventricle ejection volume. In hypovolemic patients, this decrease is even more important. After some heart beats, the decrease in right ventricle ejection volume will affect the left ventricle. The decrease in left ventricle ejection volume is proportionally identical to right ventricle ejection volume decrease and causes systolic blood pressure decrease. The decrease in systolic blood pressure, observed during the expiratory phase, may be interrupted by next respiratory cycle if respiratory rate is above 20 cycles. $\min ^{-128}$. The influence of mechanical ventilation on venous return is restricted when there is pulmonary compliance decrease or chest compliance increase ${ }^{1}$. SPV cannot be used as a left ventricular filling index in patients with pulmonary edema or under spontaneous ventilation, due to different tidal volumes observed with this type of ventilation ${ }^{6}$.

During hypovolemic shock, there is cardiac output increase and systemic vascular resistance increase. In this situation, changes in venous return determined by mechanical ventilation will be more intense. It has been shown that in sepsis patients being treated with catecholamines, SPV ddown is a major indicator of cardiac output response to volume infusion ${ }^{29}$. SPV and ddown show more important changes during absolute hypovolemia than during sodium nitroprossiate-induced arterial hypotension ${ }^{2}$. Also, SPV cannot be interpreted in the presence of arrhythmias, and nodal rhythm may increase SPV by decreasing effective preload.

Some hemodynamic parameters returned to baseline values after volume replacement, such as DPB, PAP, IRVP and ITSVE. However, some attributes remained below baseline values, such as MBP, PCWP and IRVS. Other hemodynamic parameters remained above baseline values, such as $\mathrm{CI}, \mathrm{SI}$ and ITSVD. It has to be stressed that important $\mathrm{Cl}$ increase is a characteristic of the hypertonic solution ${ }^{10}$.

SPV and ddown remained above baseline values, indicating that volume replacement with hypertonic and hyperoncontic solutions in the volume used in this study $\left(4 \mathrm{ml} . \mathrm{kg}^{-1}\right)$, has improved but not normalized volume. The fact that dogs have presented SPV consisting only of ddown after volume replacement reinforces the idea that animals where still hypovolemic.

Plasma expansion with SHD determines fast hemoglobin and blood plasma protein dilution ${ }^{30}$, as it has been observed with hemoglobin. While $7.5 \%$ sodium chloride hypertonic solution expands plasma volume by mobilizing cellular water, the association of dextran 70, a hyperoncotic colloid, increases water retention in the vessel with major plasma volume expansion ${ }^{31}$. Although the fast increase in plasma volume after SHD infusion in animals submitted to hemorrhage, blood volume remains below normal values ${ }^{17,31}$. This may explain in our study the maintenance of SPV and ddown below baseline values after SHD infusion. It has to be stressed that 
dextran 70 used in this study had a lower concentration $(3.75 \%)$ than that normally used $(6 \%)$, which may have also influenced our results.

A study on ventricular preload through SPV and transesophageal echocardiography, in 21 patients submitted to aortic surgeries and admitted to the ICU under sedation and ventilation, has shown that left ventricle size at the end of diastole was well correlated to SPV $(r=0.80)$ and ddown $(r=$ $0.83)$ range. After volume expansion, obtained in two steps with $5 \%$ human albumin $(250 \mathrm{ml})$, there has been a significant ddown linear correlation with left ventricle end diastolic area (EDa) $(r=0.63)$ and cardiac index $(\mathrm{Cl}) r=0.55)$. So, the higher the initial ddown value, the greater the EDa and Cl change after volume replacement. According to the authors, these results have shown that SPV analysis supplies valuable information about preload and left ventricle response to volume expansion ${ }^{5}$.

In our study, hypertonic solution associated to hyperoncotic solution has determined, by the effect of the first solution, a significant increase in plasma osmolality due to the increase in plasma chlorine and sodium. In general, plasma osmolality and sodium and chlorine concentration return to baseline levels 24 hours after hypertonic solution administration. Although there are no reports of adverse events, patients under hypertonic solutions need sodium levels monitoring.

In anesthetized patients submitted to hypovolemia by removing $1000 \mathrm{ml}$ of blood, it was observed that replacement with the same volume of amide has decreased SPV and ddown to values below baseline ${ }^{6}$. Several authors have observed that the association of hypertonic and hyperoncotic solutions (60 $\mathrm{mmHg}$ ) results in higher plasma volume, blood pressure and cardiac output increase, all of them dose-dependent on dex$\operatorname{tran}^{17,19,32-34}$ or amide solution ${ }^{6}$. Recently it has been shown that part of the hypertonic and hyperoncotic solution efficacy for volume expansion is due to the mobilization of intestinal proteins to the circulation in a higher proportion as compared to saline solution ${ }^{35}$.

Ddown, which during hypovolemia is virtually responsible for all SPV ${ }^{1,6}$, decreases after fluid administration (crystalloids or colloids) 1,5-7,29, while it virtually disappears during hypervolemia and/or congestive heart failure ${ }^{3}$. So, in the presence of circulatory failure, ddown may also differentiate myocardial dysfunction from inadequate preload. A high left ventricle function curve (good responsiveness to volume) is associated to an increase in ddown, while the flattening of this curve (poor responsiveness) is associated to decreased ddown ${ }^{36}$.

Similar results were obtained in sepsis and myocardial disease patients who, in response to fast volume expansion, showed a relative dup increase ${ }^{29}$. This reinforces the importance of isolated ddown and dup measurements, in addition to SPV measurements. The interpretation of SPV increase as a sign of hypovolemia when it is characterized by dup dominating ddown, may lead to unjustifiable fluid administration.

SHD infusion had side-effects, such as hyperchloremic acidosis as a consequence of hypernatremia and hyperchloremia, in addition to $\mathrm{aH}$ of approximately 5.0.The increase in $\mathrm{PaCO}_{2}$ and $\mathrm{P}_{\mathrm{ET}} \mathrm{CO}_{2}$, as a consequence of major $\mathrm{Cl}$ increase following solution infusion, has certainly also contributed to acidosis.

In conclusion, in dogs and in our experimental conditions, SPV, and especially ddown, are early hypovolemia indicators and sensitive guides for volume replacement, being better than some hemodynamic parameters, such as pulmonary capillary wedge pressure and cardiac index.

\section{REFERÊNCIAS - REFERENCES}

01. Perel A, Pizov R, Cotev S - The systolic pressure variation is a sensitive indicator of hypovolemia in ventilated dogs subjected to graded hemorrhage. Anesthesiology, 1987;67:492-502.

02. Pizov R, Ya'Ari Y, Perel A - Systolic pressure variation is greater during hemorrhage than during sodium nitroprusside induced hypotension in ventilated dogs. Anesth Analg, 1988;67: 170-174.

03. Pizov R, Ya' Ari Y, Perel A - The arterial pressure waveform during acute ventricular failure and synchronized external chest compression. Anesth Analg, 1989;65:150-156.

04. Pizov R, Segal E, Kaplan L et al - The use of systolic pressure variation in hemodynamic monitoring during deliberate hypotension in spine surgery. J Clin Anesth, 1990;2:96-100.

05. Coriat $\mathrm{P}$, Vrillon M, Perel A et al - A comparison of systolic blood pressure variations and echocardiographic estimates on end diastolic left ventricular size in patients after aortic surgery. Anesth Analg, 1994;78:46-53.

06. Rooke GA, Schwid HA, Shapira Y - The effect of graded hemorrhage and intravascular volume replacement on systolic pressure variation in humans during mechanical and spontaneous ventilation. Anesth Analg, 1995;80:925-932.

07. Ornstein E, Eidelman LA, Drenger B et al - Systolic pressure variation predicts the response to acute blood loss. J Clin Anesth, 1998;10:137-140.

08. Weiss YG, Oppeinheim-Edden A, Sprung CL et al - Systolic pressure variation in hemodynamic monitoring after severe blast injury. J Clin Anesth, 1999;11:132-135.

09. Velasco IT, Pontieri V, Rocha e Silva M et al - Hyperosmotic $\mathrm{NaCl}$ and severe hemorrhagic shock. Am J Physiol, 1980;239: H664-H673.

10. Crystal GJ, Gurevicius J, Kim SS et al - Effects of hypertonic saline solutions in the coronary circulation. Circ Shock, 1994;42:27-38.

11. Auler Jr JOC, Pereira MHC, Gomide do Amaral RV et al Hemodynamic effects of hypertonic sodium chloride during surgical treatment of aortic aneurysms. Surgery, 1987; 101:594-601.

12. Cross JS, Gruber DP, Burchard KW et al - Hypertonic saline fluid therapy following surgery: a prospective study. J Trauma, 1989;29:817-825.

13. Boldt J, Kling D, Weidler B et al - Acute preoperative hemodilution in cardiac surgery: volume replacement with a hypertonic saline - hydroxyethyl starch solution. J Cardiothor Vasc Anesth, 1991;15:23-28.

14. Järvelä K, Koskinen M, Koukinem S et al - Effects of hypertonic saline $(7.5 \%)$ on extracelular fluid volume compared with normal saline $(0.9 \%)$ and $6 \%$ hydroxyethyl starch after aortocoronary bypass graft surgery. J Cardiothor Vasc Anesth, 2001;15:210-215. 
15. Smith GJ, Kramer GC, Perron P et al - A comparison of several hypertonic solutions for resuscitation of bled sheep. J Surg Res, 1985;35:517-528.

16. Rocha e Silva M - Hypertonic saline resuscitation: a new concept. Baillière's Clin Anaesthesiol, 1997;11:127-142.

17. Velasco IT, Rocha e Silva M, Oliveira MA et al - Hypertonic and hyperoncotic resuscitation from severe hemorrhagic shock in dogs: a comparative study. Crit Care Med, 1989;17:261-264.

18. Kramer GC, Walsh JC, Perron PR et al - Comparison of hypertonic saline dextran versus hypertonic saline hetastarch for resuscitation of hipovolemia. Braz J Med Biol Res, 1989;22: 279-282.

19. Christ AT, Niklas M, Kreimeir V et al - Hyperosmotichyperoncotic solutions during abdominal aortic aneurysm (AAA) resection. Acta Anaesthesiol Scand, 1997;41:62-70.

20. Kreimeir V, Brückner UB, Wiemczy KS et al - Hyperosmotic saline dextran for resuscitation from traumatic-hemorrhagic hypotension: effect on regional blood flow. Circ Shock 1990;32: 83-99.

21. Nolte D, Bayer M, Lehr HA et al - Attenuation of postischemic microvascular disturbances in striate muscle by hyperosmolar saline dextran. Am J Physiol, 1992;263:H1411-1416.

22. Haskins SC - Monitoring the Anesthetized Patient, em: Thurmon JC, Tranquilli WJ, Benson GJ - Lumb \& Jones Veterinary Anesthesia, $3^{\text {rd }}$ Ed, Baltimore, Willians \& Wilkins, 1996;409-424.

23. Committee on Trauma, American College of Surgeons - The advanced trauma life support: courses for physicians. Chicago, 1993;75-94.

24. Rocha e Silva M, Poli de Figueiredo LF - Fisiopatologia do Choque Hipovolêmico, em: Rocha e Silva M - Fisiopatologia Cardiovascular. São Paulo, Atheneu, 2000;155-172.

25. Hansen RW, Viquerat CE, Mattay MA et al - Poor correlation between pulmonary arterial wedge pressure and left ventricular and diastolic volume after coronary artery bypass surgery. Anesthesiology, 1986;67:764-770.

26. Rapper R, Sibbald WJ - Misled by the wedge. The Swan-Ganz catheter and LV preload. Chest, 1986;88:427-434.

27. Marik PE - The systolic blood pressure variation as an indicator of pulmonary capillary wedge pressure in ventilated patients. Anaesth Intensive Care, 1993;21:405-408.

28. Coriat $P$ - Detecção de Hipovolemia no Intra-Operatório, em: Braz JRC, Auler Jr JOC, Amaral JLG, et al - O Sistema Cardiovascular e a Anestesia. São Paulo, UNESP \& Artes Médicas, 1997;119-124.

29. Tavernier B, Makhotine O, Lebuffer G et al - Systolic pressure variation as a guide to fluid therapy in patients with sepsis-induced hypotension. Anesthesiology, 1998;89:1313-1321.

30. Wolf MB, Watson PD - Measurement of osmotic reflexion for small molecular in cats hindlimbs. Am J Physiol, 1989;256: $\mathrm{H} 282-\mathrm{H} 290$.

31. Smith GJ, Kramer GC, Perron P et al - A comparison of several hypertonic solutions for resuscitation of bled sheep. J Surg Res, 1985;35:517-528.

32. Walsh JC, Kramer GC - Resuscitation of hypovolemic sheep with hypertonic saline/dextran: the role of dextran. Circ Shock, 1991;34:336-343.

33. Vassar MJ, Perry CA, Holcroft JW - Prehospital resuscitation of trauma patients with $7.5 \mathrm{NaCl}$ versus $7.5 \mathrm{NaCl}$ added dextran: a controlled trial. J Trauma, 1993;34:622-632.

34. Oliveira SA, Bueno RM, Souza JM et al - Effects of hypertonic solution saline dextran on the postoperative evolution of Jehovah's Witness patients submitted to cardiac surgery with cardiopulmonary bypass. Shock, 1995;3:391-394.
35. Kramer GC, Vane LA, Kilicturgay $N$ et al - Resuscitation with hypertonic saline dextran mobilizes interstitial protein more effectively than normal saline. Anesthesiology, 1999;91:(Suppl3): A696.

36. Perel A - Assessing fluid responsiveness by the systolic pressure variation in mechanically ventilated patient. Anesthesiology, 1998;89:1309-1310.

\section{RESUMEN}

Paiva Filho O, Braz JRC, Silva FP, Pedro TO, Nascimento Jr P Variación de la Presión Sistólica como Indicadora Precoz de Hipovolemia y Guía de Reposición Volemica con Solución Hiperosmótica e Hiperoncótica en el Perro

JUSTIFICATIVA Y OBJETIVOS: Estudios introdujeron un nuevo método para evaluación de la pré-carga, baseados en la análisis de la variación de la presión sistólica (VPS) durante ventilación artificial. El objetivo de esta investigación es evaluar se la VPS y la derivada delta down (ddown) son indicadoras precoces de hipovolemia y guías de reposición volémica con solución hiperosmótica e hiperoncótica.

MÉTODO: Doce perros fueron sometidos a sangramientos parciales de $5 \%$ de la volemia hasta llegar a $20 \%$ de la volemia $\left(14 \mathrm{ml} . \mathrm{kg}^{-1}\right)$. Antes (control) y después de cada sangramiento fueron realizadas análisis hemodinámicas, respiratorias y sanguíneas. Después, los perros fueron sometidos a la reposición con solución de $\mathrm{NaCl}$ a 7,5\% en dextran 70 a 3,75\% (SHD) $\left(4 \mathrm{ml} . \mathrm{kg}^{-1}\right)$ y nuevas análisis de los atributos estudiados fueron realizadas a los 5 y 30 min después de la reposición.

RESULTADOS: La presión arterial media diminuyó durante el sangramiento y aumentó después de la reposición, sin retornar a los valores del control. Las presiones de la arteria pulmonary del átrio derecho (PAD) diminuyeron antes y aumentaron después de la reposición para valores semejantes a los del control. La presión de la arteria pulmonar ocluida (PAPO) diminuyó después del primero sangramiento y se mantuvo en los valores abajo a los del control mismo después de la reposición. El índice cardíaco no se alteró, más, aumentó después de la reposición, para valores superiores a los del control. El índice sistólico (IS) diminuyó antes y aumentó después de la reposición, en niveles superiores a los del control. Los indices de resistencia vascular sistemica (IRVS) y pulmonar (IRVP) no se alteraran antes, más diminuyeron después de la reposición, con el IRVS en niveles inferiores a los del control y el IRVP en niveles semejantes a los do control. Los índices de trabajo sistólico de los ventrículos derecho (ITSVD) e izquierdo (ITSVE) diminuían durante el sangramiento, más aumentaron después de la reposición, con el ITSVD en niveles superiores a los del control y el ITSVE en niveles semejantes a los del control. La VPS y ddown aumentaron progresivamente durante el sangramiento y diminuyeron después de la reposición, mas manteniendo en valores superiores a los del control. Las mayores correlaciones de VPS y ddown fueron con IS, PAPO, PAD Y ITSVE.

CONCLUSIONES: En el perro, en las condiciones utilizadas, la VPS y su derivada ddown son indicadoras precoces de hipovolemia y guías sensibles de reposición volémica con $S H D$. 